\title{
Dysfunctional SEMA3E signaling underlies gonadotropin-releasing hormone neuron deficiency in Kallmann syndrome
}

\author{
Anna Cariboni, ${ }^{1,2}$ Valentina André, ${ }^{2}$ Sophie Chauvet, ${ }^{3}$ Daniele Cassatella, ${ }^{4}$ Kathryn Davidson, ${ }^{1}$ Alessia Caramello, ${ }^{1}$ \\ Alessandro Fantin, ${ }^{1}$ Pierre Bouloux, ${ }^{5}$ Fanny Mann, ${ }^{3}$ and Christiana Ruhrberg ${ }^{1}$ \\ 'UCL Institute of Ophthalmology, University College London, London, United Kingdom. 'University of Milan, Department of Pharmacological and Biomolecular Sciences, Milan, Italy. \\ ${ }^{3}$ Aix-Marseille Université, CNRS, Marseille, France. ${ }^{4}$ Centre Hospitalier Universitaire Vaudois, Service of Endocrinology, Diabetes and Metabolism, Lausanne, Switzerland. \\ ${ }^{5}$ Centre for Neuroendocrinology, UCL Medical School, University College London, London, United Kingdom.
}

Individuals with an inherited deficiency in gonadotropin-releasing hormone (GnRH) have impaired sexual reproduction. Previous genetic linkage studies and sequencing of plausible gene candidates have identified mutations associated with inherited GnRH deficiency, but the small number of affected families and limited success in validating candidates have impeded genetic diagnoses for most patients. Using a combination of exome sequencing and computational modeling, we have identified a shared point mutation in semaphorin 3E (SEMA3E) in 2 brothers with Kallmann syndrome (KS), which causes inherited GnRH deficiency. Recombinant wild-type SEMA3E protected maturing GnRH neurons from cell death by triggering a plexin D1-dependent (PLXND1-dependent) activation of PI3K-mediated survival signaling. In contrast, recombinant SEMA3E carrying the KS-associated mutation did not protect GnRH neurons from death. In murine models, lack of either SEMA3E or PLXND1 increased apoptosis of GnRH neurons in the developing brain, reducing innervation of the adult median eminence by $\mathrm{GnRH}$-positive neurites. GnRH neuron deficiency in male mice was accompanied by impaired testes growth, a characteristic feature of KS. Together, these results identify SEMA3E as an essential gene for GnRH neuron development, uncover a neurotrophic function for SEMA3E in the developing brain, and elucidate SEMA3E/PLXND1/PI3K signaling as a mechanism that prevents GnRH neuron deficiency.

\section{Introduction}

In mammals, fertility depends on the timely onset and cyclic secretion of the neurohormone gonadotropin-releasing hormone $(\mathrm{GnRH})$, which is secreted into the portal blood vessels of the pituitary gland by approximately 1,000 hypothalamic neurons (1). Deficiency in hypothalamic GnRH impairs puberty and almost invariably leads to infertility, causing a genetic condition that is known as hypogonadotropic hypogonadism $(\mathrm{HH})$ or, when combined with anosmia, as Kallmann syndrome (KS) (2). The previous analysis of a single human fetus from a family with chromosome X-linked KS (3), combined with the analysis of mouse models with engineered genetic mutations, shows that defective $\mathrm{GnRH}$ neuron development can cause $\mathrm{HH} / \mathrm{KS}$ (4). For example, we recently demonstrated that the class 3 semaphorin SEMA3A is essential for axon guidance during GnRH neuron development in mice (5), and others subsequently identified mutations in SEMA3A in patients with KS $(6,7)$. These and other studies (8) show that genetic mouse models are valuable tools to identify candidate genes for $\mathrm{HH} / \mathrm{KS}$ and elucidate how their mutation

\section{Related Commentary: p. 2275}

Conflict of interest: The authors have declared that no conflict of interest exists. Submitted: August 29, 2014; Accepted: April 2, 2015.

Reference information: J Clin Invest. 2015;125(6):2413-2428. doi:10.1172/JCI78448. may compromise the formation of the GnRH neuron system in patients for whom the diagnosis of impaired GnRH secretion alone provides little insight into the disease mechanism (1). However, mutations have so far only been identified in approximately $40 \%$ of $\mathrm{HH} / \mathrm{KS}$ cases, partly because the small number of families affected by this disease impedes genetic linkage studies, but also because sporadic mutations cannot be identified with linkage studies, and some cases are probably oligogenic (8).

Here, we have combined exome sequencing of KS patients with bioinformatics, functional in vitro studies, and the phenotyping of genetically modified mice to identify SEMA3E as a gene that is essential for $\mathrm{GnRH}$ neuron development and that is affected in KS. The SEMA3E gene encodes a secreted class 3 semaphorin that triggers repulsion of endothelial cells in specific vascular beds $(9,10)$ and modulates axonal growth and synaptic connectivity for the correct wiring of the CNS (11-13). Unexpectedly, we found that the $S E M A 3 E$ mutation, rather than affecting axon guidance in the GnRH neuron system, instead compromised survival signaling in $\mathrm{GnRH}$ neurons via PI3 kinase. Accordingly, loss of SEMA3E or its receptor plexin D1 (PLXND1) in mice caused GnRH neuron apoptosis during the developmental window in which these neurons express PLXND1 and migrate through SEMA3E-expressing brain regions to reach the hypothalamus. SEMA3E-mediated survival signaling also relied on KDR, a receptor tyrosine kinase that was 
A

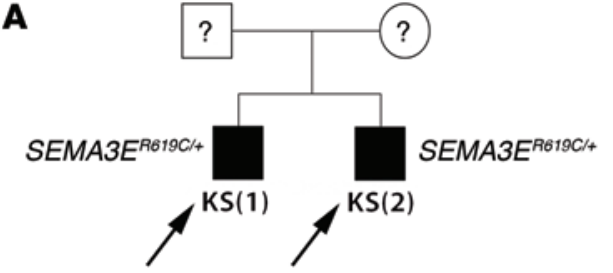

C

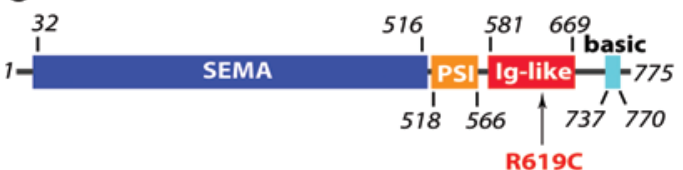

B

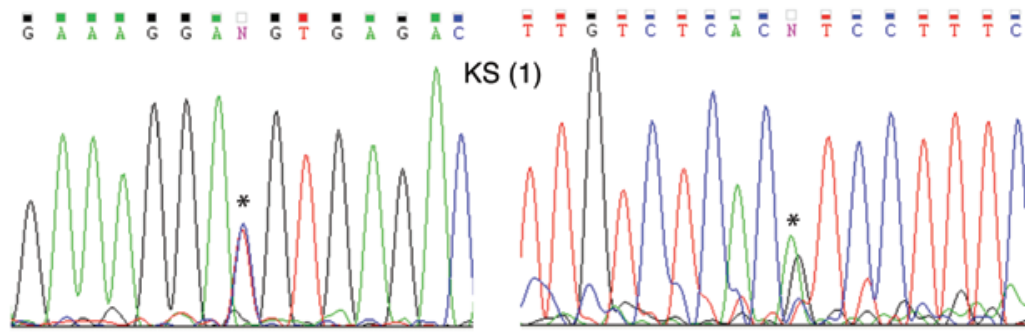

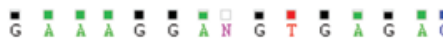

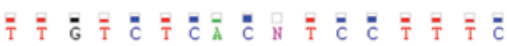
KS (2)
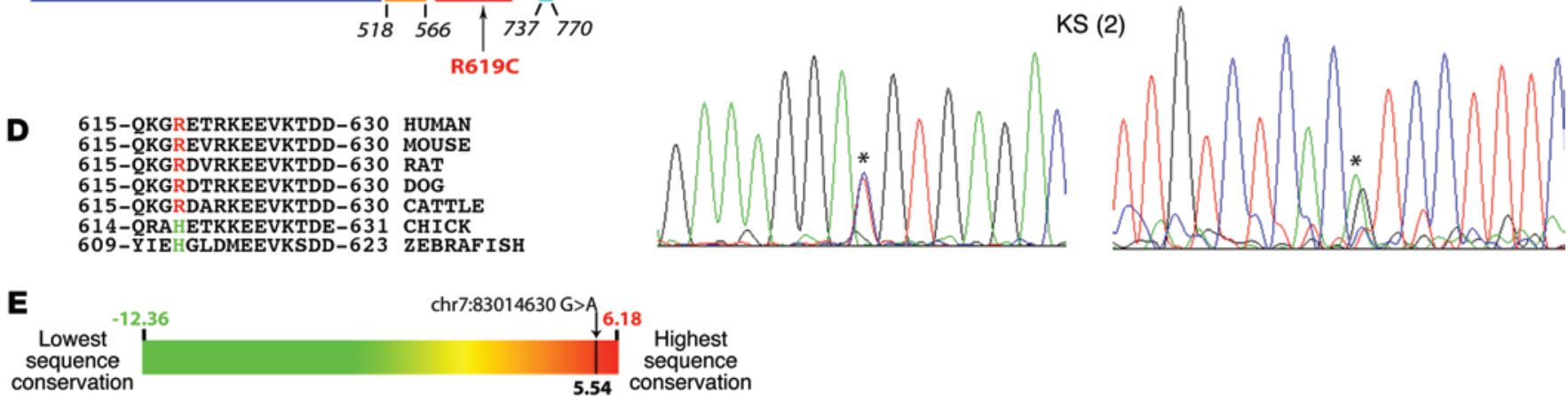

F
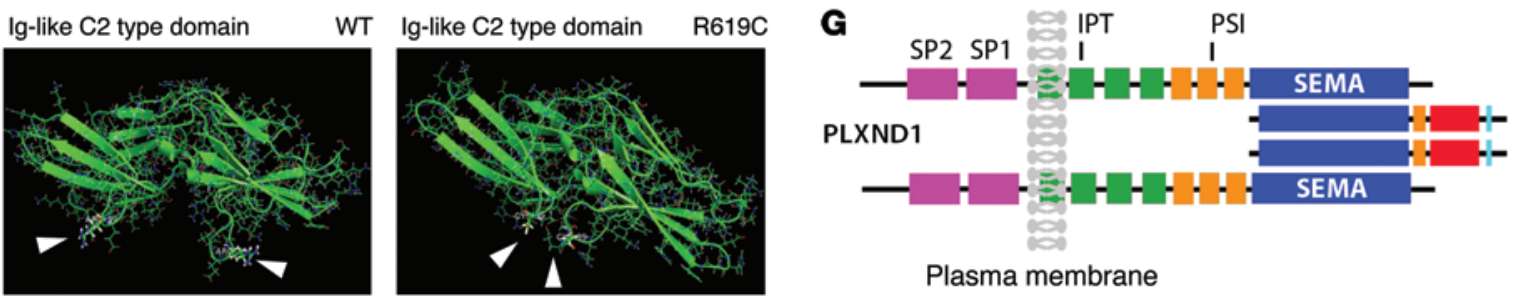

SEMA3E

Figure 1. Exome sequencing identifies a SEMA3E point mutation in KS patients. (A) Pedigree of brothers affected by KS carrying a novel SEMA3E mutation. Circle denotes female; square denotes male; black square denotes affected male; arrows indicate the probands. (B) Sequence chromatograms of nucleotides 1849-1863 of the SEMA3E coding sequence in 2 brothers carrying a nucleotide substitution in exon 16 (left side, forward strand; right side, reverse strand; the positions of the $C>T$ and corresponding $G>A$ change are indicated with asterisks). (C) Diagram of the SEMA3E functional domains: SEMA, PSI (plexin/semaphorin/integrin), Ig (Ig-like, C2-type), and basic domains; their position and the position of the mutated amino acid residue within the protein sequence are indicated. (D) Alignment of partial protein sequences of vertebrate SEMA3E orthologs shows that the R619 residue is evolutionarily conserved in mammals (red), but not in chick or zebrafish (green). (E) Genomic evolutionary rate profiling of sequence constraint for the SEMA3E mutation in the 2 KS brothers using GERP++ analysis provided an RS score of 5.54, which is close to the maximum score of 6.18 for complete conservation across all mammalian species. (F) Computational models of a dimer of the Ig domain of WT and mutant SEMA3E. The model is based on the Robetta algorithm for comparative protein structure prediction. The highest-scoring dimer model is shown. The R619 residue and the R619C substitution are indicated by white arrowheads. (C) Schematic drawing illustrating the protein interaction between SEMA3E and PLXND1, including its structural domains - the SEMA and PSI domains, similar to those of SEMA3E - as well as the IPT (Ig-like, plexin, transcription factor) domains and the serine/threonine protein kinase catalytic domains 1 and 2 (SP1 and SP2).

previously implicated as a coreceptor for PLXND1 in SEMA3Emediated axonal growth (11). Thus, our study has uncovered a novel role for SEMA3E as a neurotrophic factor that delivers essential survival signals for GnRH neurons via PLXND1 and KDR. Finally, we show that Sema3e interacts genetically with
Chd7, a gene previously implicated in KS. Our study further provides proof of principle that exome sequencing, when combined with suitable in silico, in vitro, and in vivo animal models, can identify genetic pathways involved in GnRH neurodevelopment and deficiency.

Table 1. Chromosome position, nucleotide substitution, amino acid substitution, and bioinformatic predictions of the identified SEMA3E and CHD7 missense mutations

$\begin{array}{lllllll}\text { Chr } & \text { Position } & \text { Nt sub } & \text { AA sub } & \text { PolyPhen-2 } & \text { SIFT } & \text { MutationTaster } \\ 7 & 830114630 & \text { G }>\text { A } & \text { R619C } & \text { Possibly damaging } & \text { Deleterious } & \text { Disease causing } \\ 8 & 61735160 & \text { T }>\text { G } & \text { F1019C } & \text { Possibly damaging } & \text { Deleterious } & \text { Disease causing }\end{array}$

AA sub, amino acid substitution; Chr, chromosome; Nt sub, nucleotide substitution. PolyPhen-2: http://genetics.bwh.harvard.edu/pph2/; SIFT: http://sift. jcvi.org/; MutationTaster: http://www.mutationtaster.org/. 
A
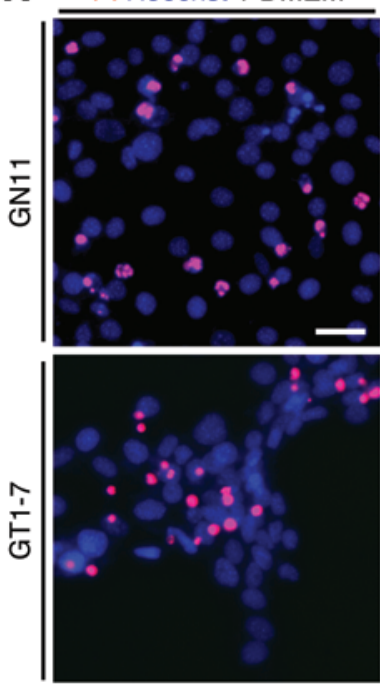

C

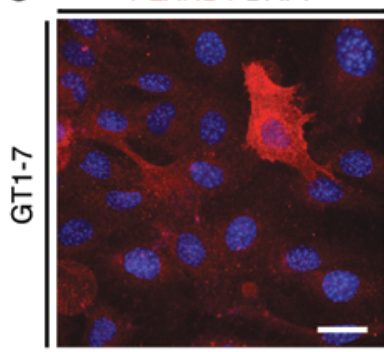

E

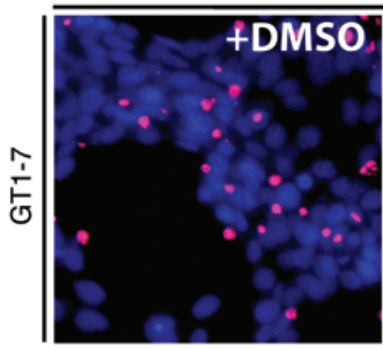

$\mathbf{F}$

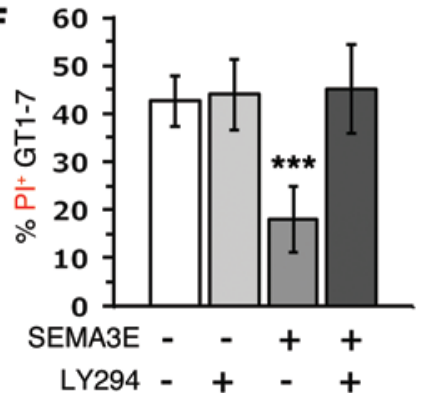

PI Hoechst + FBS
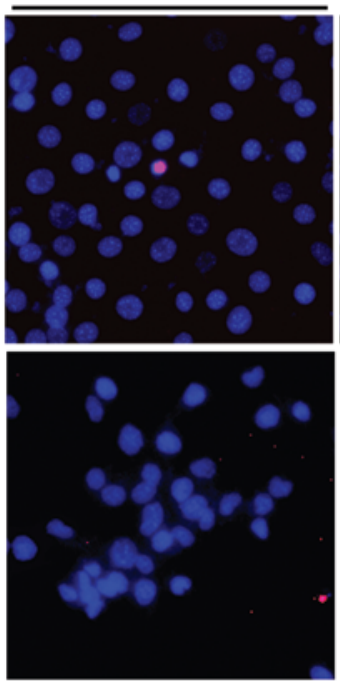

PI Hoechst + SEMA3E
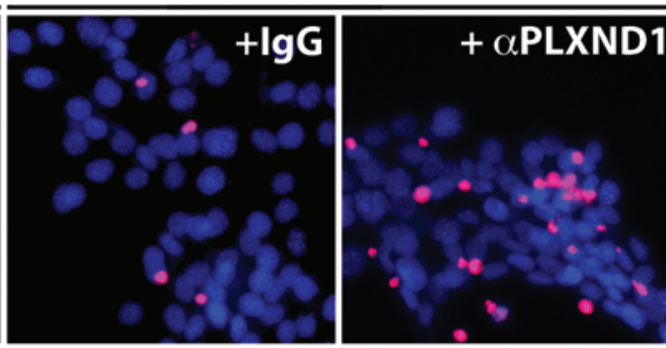
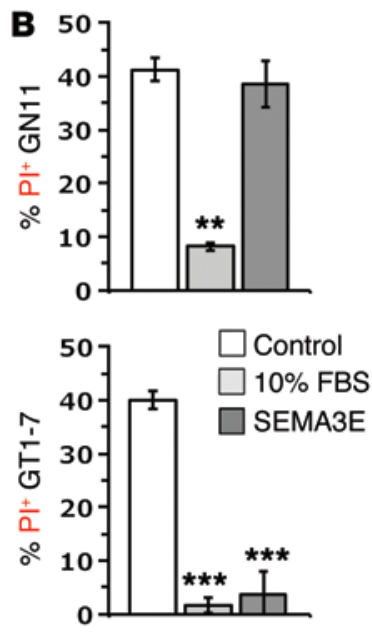

PI Hoechst + SEMA3E
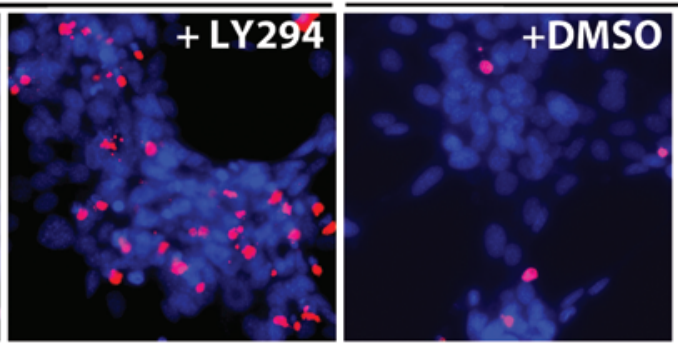

G

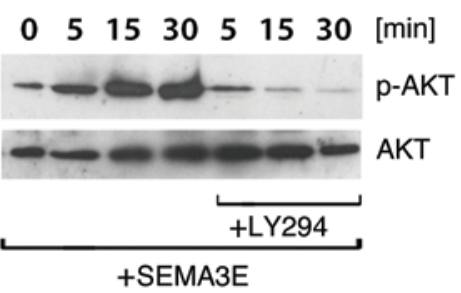

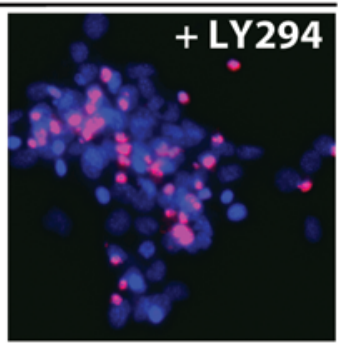

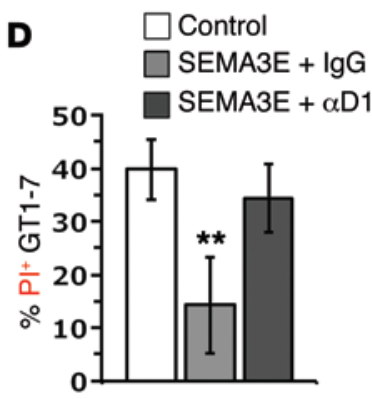

H

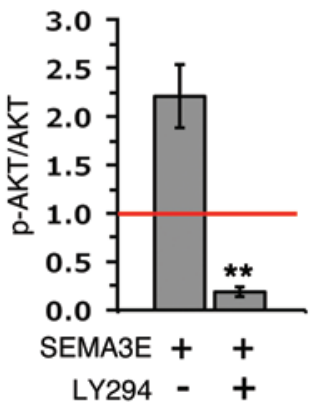

Figure 2. SEMA3E promotes GnRH neuron survival via PLXND1 and PI3 kinase activation. (A and B) SEMA3E protects GT1-7 but not GN11 cells from death induced by serum starvation. Serum-starved GN11 (top row) and GT1-7 (bottom row) cells were treated with serum or SEMA3E. Dying cells were visualized by PI staining in the presence of Hoechst nuclear counterstain (A) to determine the proportion of PI-positive cells relative to all Hoechst-stained cells, shown as the mean \pm SEM; $n=3$; ${ }^{*} P<0.01$ and ${ }^{* *} P<0.001$ by 1 -way ANOVA (B). (C and D) Blocking PLXND1 abolished SEMA3E-mediated neuroprotection of GT1-7 cells. Immunofluorescence staining with PLXND1 antibody ( $\alpha$ PLXND1) showed that GT1-7 cells expressed PLXND1 (left panel, C). PI and Hoechst staining showed that $\alpha$ PLXND1, but not control IgG, inhibited SEMA3E-mediated neuroprotection in GT1-7 cells (right 2 panels, C). Proportion of PI-positive cells in all Hoechst-stained cells (D), shown as the mean \pm SEM; $n=3$; ${ }^{*} P<0.01$ by 1-way ANOVA. (E and F) Blocking PI3K abolished SEMA3E-mediated neuroprotection of GT1-7 cells. PI and Hoechst staining showed that LY294 did not affect cell death under control conditions (left 2 panels, E), but inhibited SEMA3E-mediated neuroprotection in GT1-7 cells (right 2 panels, E). Proportion of PI-positive cells in all Hoechst-stained cells (F), shown as the mean \pm SEM. $n=3$; ${ }^{* *} P<0.001$ by 1 -way ANOVA. (G and H) SEMA3E promoted PI3K-dependent AKT phosphorylation in GT1-7 neurons. Immunoblotting shows increased levels of phosphorylated AKT ( $p$-AKT) (Ser473) relative to total AKT in serum-starved GT1-7 cells treated with SEMA3E, and LY294 abolished SEMA3E-induced AKT phosphorylation. $n=3$; ${ }^{* *} P<0.01$ by Student's $t$ test. Scale bars: $10 \mu \mathrm{m}$ (left panel, C); $25 \mu \mathrm{m}$ (all other panels). The red line in $\mathbf{H}$ indicates the $\mathrm{p}-\mathrm{AKT} / \mathrm{AKT}$ ratio in untreated serum-starved GT1-7 cells, which was set to 1. 
A
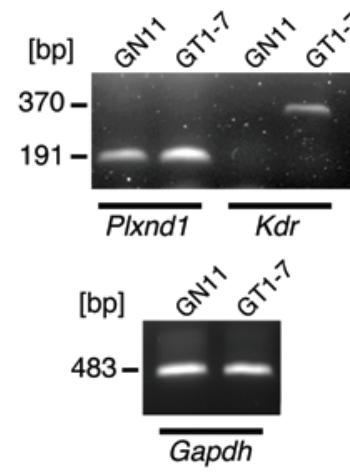

D

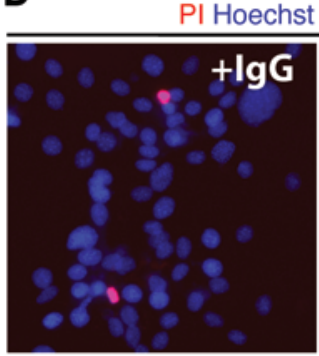

B

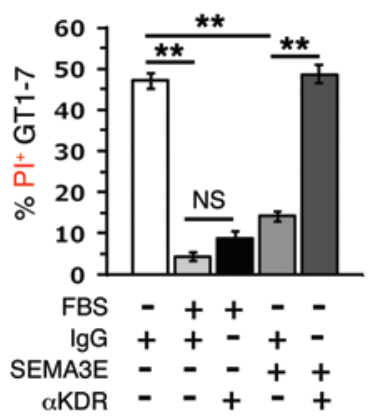

C

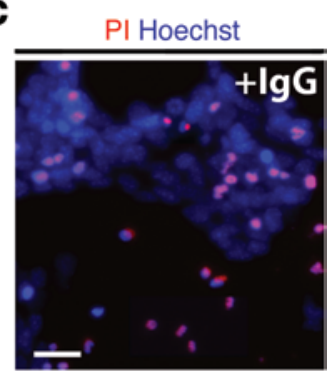

Figure 3. KDR is important for SEMA3E-induced GnRH neuron survival in vitro. (A) Expression of KDR in GT1-7 cells. RT-PCR analysis revealed expression of PIxnd1 in GN11 cells and, at higher levels, in GT1-7 cells, while $K d r$ was detectably expressed only in GT1-7 cells; Gapdh served as a loading control. (B-D) Loss of KDR function impaired the SEMA3E-mediated neuroprotection of GT1-7 cells. Quantification of cell death in serumstarved GT1-7 cultures, expressed as a percentage of PI-positive cells in all cells, which were identified by Hoechst staining (B; $n=3 ;{ }^{* *} P<0.01$ by 1 -way ANOVA), and representative examples of stained cultures treated with control IgG in the absence of neurotrophic factors (C) or in the presence of FBS or SEMA3E with control IgG or $\alpha$ KDR (D). Scale bar: $25 \mu \mathrm{m}$ in C.

\section{Results}

A point mutation in the SEMA3E gene in 2 brothers with KS. By performing exome sequencing of patients with $\mathrm{KS}$, we identified 2 male siblings (Figure 1A) with a heterozygous substitution of nucleotide 1855 in the SEMA3E coding sequence (ENST00000307792.3:c.1855C >T) (Figure 1B). The brothers had been diagnosed at ages 15 and 17, respectively, with anosmia $\left(<10^{\text {th }}\right.$ percentile on a standardized smell identification test), prepubertal testes ( $<2 \mathrm{ml}$; normally $>20 \mathrm{ml})$, and GnRH deficiency (revealed by measuring the levels of the gonadotropin LH, which were $1.0 \mathrm{IU} / \mathrm{l}$ plasma, and are normally $>1.5 \mathrm{IU} / \mathrm{l}$ ) in the setting of hypogonadal testosterone levels ( $3.5 \mathrm{nmol} / 1$ plasma; normally $10-50 \mathrm{nmol} / \mathrm{l})$. The mutation was not present in the 1000 Genomes database and occurred with a very low minor allele frequency in the NHLBI GO Exome Sequencing Project (ESP) database in European ancestry samples (0.0004\%). The c.1855C $>$ T mutation substitutes a charged arginine residue with a neutral but potentially disulfide-bondable cysteine residue (p.R619C) and is predicted to be deleterious, possibly damaging, and disease causing according to the SIFT (14), PolyPhen-2 (15), and MutationTaster (16) bioinformatic tools, respectively (Table 1). The R619C mutation resides in the extracellular, C-terminal_Ig-like_C2-type domain of SEMA3E (Figure 1C) and affects an arginine residue that is highly conserved between mammals, but not lower vertebrate species (Figure 1D). Genomic evolutionary rate profiling (GERP++) of a large collection of mammalian species provided a rejected substitution (RS) score that is close to the maximum estimated RS score (17), consistent with strong evolutionary constraint against the p.R619C mutation in mammals (Figure 1E).

Because the precise structure of the SEMA3E_Ig domain has not yet been determined through crystallography, we predicted its structure by using the Robetta algorithm (18) to align it with the corresponding domains from SEMA3A and other similar entries in the Research Collaboratory for Structural Bioinformatics (RSCB) Protein Data Bank (PDB). This analysis suggested that the R619C substitution subtly alters the domain conformation of the SEMA3E dimer and places the novel cysteine residues in close apposition (Figure 1F). The mutation does not reside in a domain predicted to mediate binding of SEMA3E to its receptor PLXND1 (Figure 1G). Because the specific role of the SEMA3E_Ig domain and the impact of the p.R619C mutation on its function is unknown, we compared the activity of the wild-type (WT) and mutant recombinant protein in tissue culture studies and used mouse genetic tools to examine the requirement for SEMA3E in the GnRH neuron system.

SEMA3E signals through PLXND1 in GnRH neurons to promote their survival by activating PI3K signaling. To understand how the SEMA3E p.R619C mutation contributes to KS, we defined the cellular and biochemical mechanisms through which SEMA3E normally affects GnRH neurons. For these experiments, we used GN11 and GT1-7 cells as established models of immature migrating and maturing hypothalamic GnRH neurons, respectively (19). Prior work had already validated these neuronal cells as useful tools to identify molecular pathways involved in $\mathrm{GnRH}$ neuron development and adult physiology, because they overcome the difficulty in isolating primary GnRH neurons in sufficient quantities for biochemical and cell biological assays due to their low numbers and scattered distribution throughout the nose and brain (5,20-22). We first asked whether SEMA3E affected GN11 neuron migration in a Transwell assay previously used to identify guidance cues for GnRH neurons $(23,24)$, but did not observe any effect (mean number of migrated cells $/ \mathrm{mm}^{2} \pm$ SEM: control, $711 \pm 21.5$ vs. SEMA3E, $678 \pm 15.4 ; P>0.05)$. Because SEMA3E promotes cancer cell survival (25), we next asked whether it is neuroprotective for GN11 cells, which were previously shown to use VEGF as a survival signal (21). However, SEMA3E was unable to protect GN11 neurons from death; thus, $40 \%$ of cells were propidium iodide (PI) positive after serum withdrawal, whereby the reintroduction of serum, but not recombinant SEMA3E, for the final 12 hours of the assay suppressed cell death (Figure 2, A and B; proportion of $\mathrm{PI}^{+}$cells, mean \pm SEM: GN11: DMEM, $41.3 \% \pm 1.3 \%$ vs. $10 \%$ 

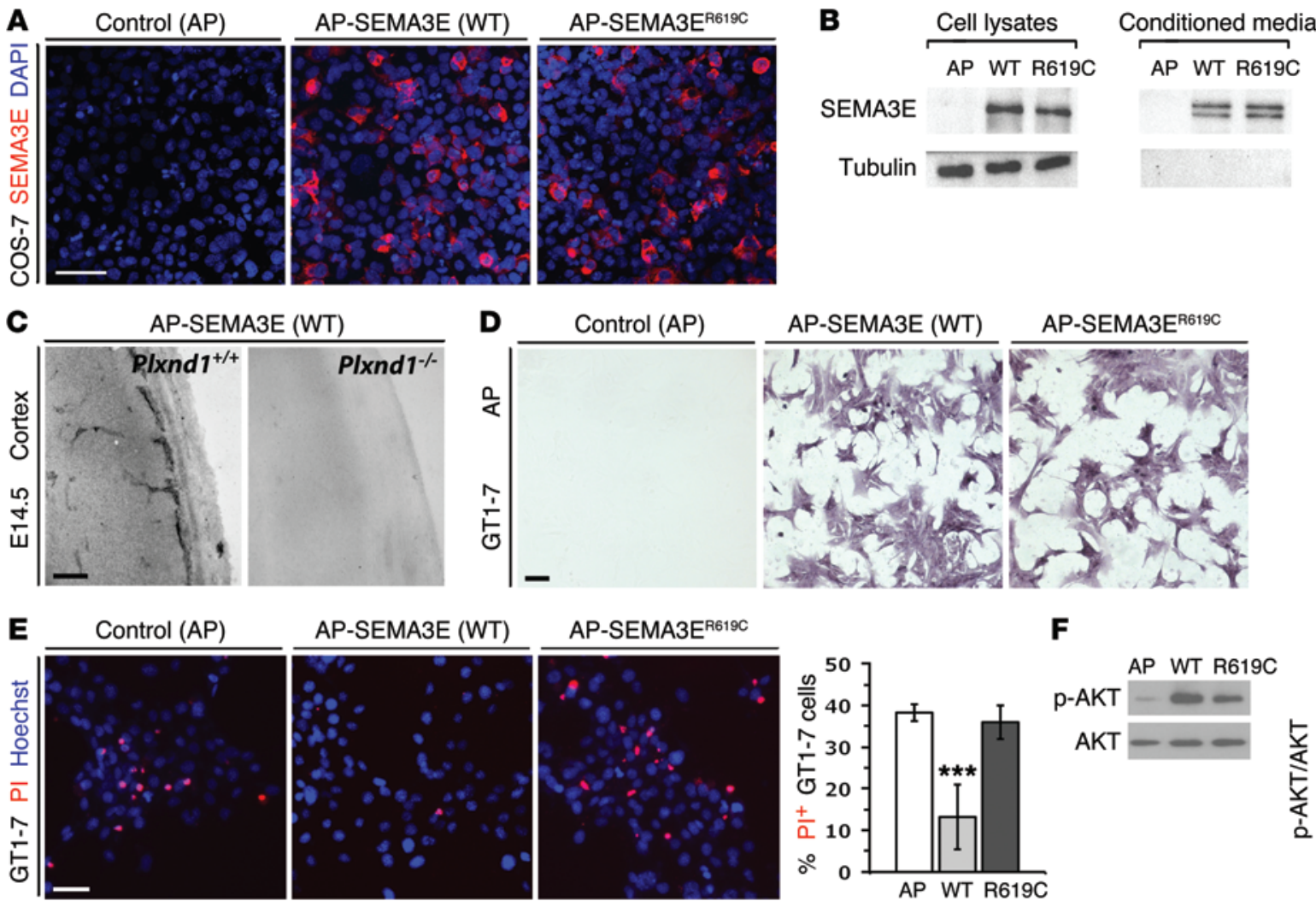

AP-SEMA3ER619C

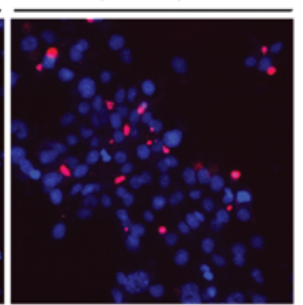

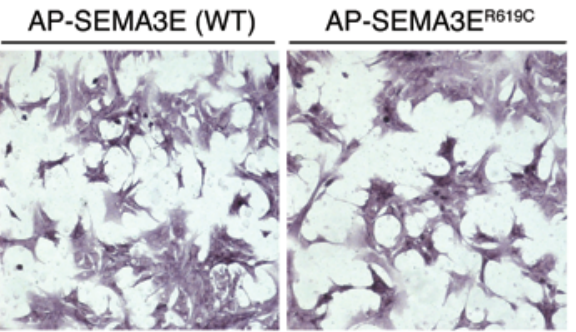

$\mathbf{F}$
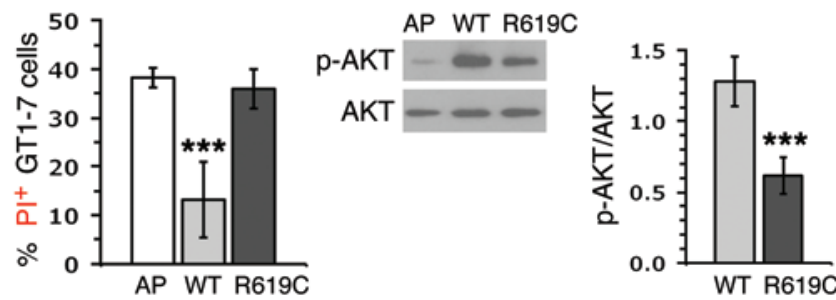

Figure 4. The SEMA3E ${ }^{\mathrm{R} 619 \mathrm{C}}$ mutation fails to rescue GT1-7 neuron survival. (A and B) Generation of WT and mutant SEMA3E AP fusion proteins. COS-7 cells were transfected with a control expression vector or vectors encoding AP fused to human SEMA3E or mutant SEMA3ER619. Cells were immunolabeled (A) or immunoblotted (B) with an antibody against SEMA3E. Cells and conditioned media contained a band of approximately 180 kDa, composed of the 95-kDa hSEMA3E and 85-kDa AP fragments. Tubulin was used as a loading control. (C) AP-SEMA3E bound WT, but not PIxnd1-null, tissue. Sagittal sections of E14.5 mouse cerebral cortex of the indicated genotypes were incubated with AP-SEMA3E. Note that SEMA3E bound blood vessels in WT, but not in Plxnd1-null, tissue. (D) AP-SEMA3E binding to GnRH neurons in vitro. The incubation of GT1-7 cells with COS cell-conditioned DMEM containing AP ligands demonstrated similar binding of WT AP-SEMA3E and mutant AP-SEMA3ER619 to CT1-7 cells, while AP alone did not bind the cells. (E) SEMA3E ${ }^{\text {R619c }}$ was not neuroprotective for GT1-7 cells. PI and Hoechst staining shows that WT SEMA3E, but not SEMA3ER619C, mediated neuroprotection of serumstarved GT1-7 cells. The percentage of PI-positive cells relative to Hoechst-stained cells is shown as the mean \pm SEM. $n=3$; ${ }^{* *} P<0.001$ by $1-$ way ANOVA. (F) The SEMA3E ${ }^{\mathrm{R} 619 \mathrm{C}}$ mutation impaired PI3K-dependent AKT phosphorylation. Immunoblotting and graph quantitation show AKT (Ser473) phosphorylation relative to total AKT in serum-starved GT1-7 cells treated for 15 minutes with WT SEMA3E or SEMA3ER619 $. n=3$; ${ }^{* * *} P<0.001$ by Student's $t$ test. Scale bars: $50 \mu \mathrm{m}$ (A), $150 \mu \mathrm{m}$ (C), $20 \mu \mathrm{m}$ (D), $100 \mu \mathrm{m}$ (E).

FBS, $8.2 \% \pm 0.4 \%, P<0.01$; DMEM vs. SEMA3E, $38.9 \% \pm 2.2 \%$, $P>0.05)$. In contrast, cell death of GT1-7 cells, a model of maturing GnRH neurons, was dramatically reduced by SEMA3E; thus, $40 \%$ of cells were PI positive after serum withdrawal, but serum or recombinant SEMA3E for the final 12 hours suppressed cell death (Figure 2, A and B; proportion of $\mathrm{PI}^{+}$cells, mean \pm SEM: GT1-7: DMEM, $40.1 \%$ $\pm 0.9 \%$; $10 \%$ FBS, $1.6 \% \pm 0.8 \%$; SEMA3E, $3.6 \% \pm 2.2 \%$; $P<0.001$, DMEM vs. $10 \%$ FBS or SEMA3E). These observations predict a survival function for SEMA3E in hypothalamic GnRH neurons.

Because SEMA3E signals through PLXND1, we examined whether PLXND1 mediated SEMA3E-dependent neuroprotection of GT1-7 cells. Immunofluorescence staining with a PLXND1 antibody that fails to stain Plxnd1-null mouse tissue (see mouseKO analysis below) showed that GT1-7 cells expressed PLXND1 (Figure 2C). Because this antibody can block PLXND1 function (26), we also used it to treat GT1-7 cells and found that it abolished the protective effect of SEMA3E after serum withdrawal (Figure 2, C and D; proportion of $\mathrm{PI}^{+}$cells, mean \pm SEM: DMEM, $39.8 \%$ $\pm 5.7 \%$; SEMA3E + IgG, $14.3 \% \pm 9.1 \%$; SEMA3E + $\alpha$ PLXND1,
$34.4 \% \pm 6.4 \% ; P<0.01$, SEMA3E + IgG vs. DMEM or SEMA3E + PLXND1). Excluding unspecific effects of the antibody on cell survival, we observed that blocking PLXND1 function did not affect GN11 cells, which use VEGF (21), but not SEMA3E, for survival signaling (proportion of $\mathrm{PI}^{+}$cells, mean \pm SEM: DMEM, 38.9\% \pm $11.2 \%$; SEMA3E + IgG, $41.1 \% \pm 9.5 \%$; SEMA3E + $\alpha$ PLXND1, $38.9 \%$ $\pm 7.4 \%)$. Together, these findings support the idea that SEMA3E promotes the survival of maturing hypothalamic, but not immature, GnRH neurons in a PLXND1-dependent fashion.

Activation of PI3K and its effector AKT plays a key role in survival signaling in many cell types, including neurons (27). In agreement with this, treatment with the PI3K inhibitor LY294 prevented the SEMA3E-mediated protection of GT1-7 cells after serum withdrawal (Figure 2, E and F; DMSO, 42.6\% $\pm 2.6 \%$; LY294, 43.9\% $\pm 3.3 \%$; SEMA3E + DMSO, $17.9 \% \pm 3.1 \%$; SEMA3E + LY194, 45.1\% $\pm 4.6 ; P<0.001$, SEMA3E + DMSO vs. DMSO) and abrogated AKT activation upon SEMA3E stimulation (Figure 2, G and $\mathrm{H}$; relative AKT activation at $15 \mathrm{~min}$ : SEMA3E, $2.209 \pm 0.3$ and SEMA3E + LY294, 0.19 \pm 0.05). 
PLXND1 GnRH DAPI
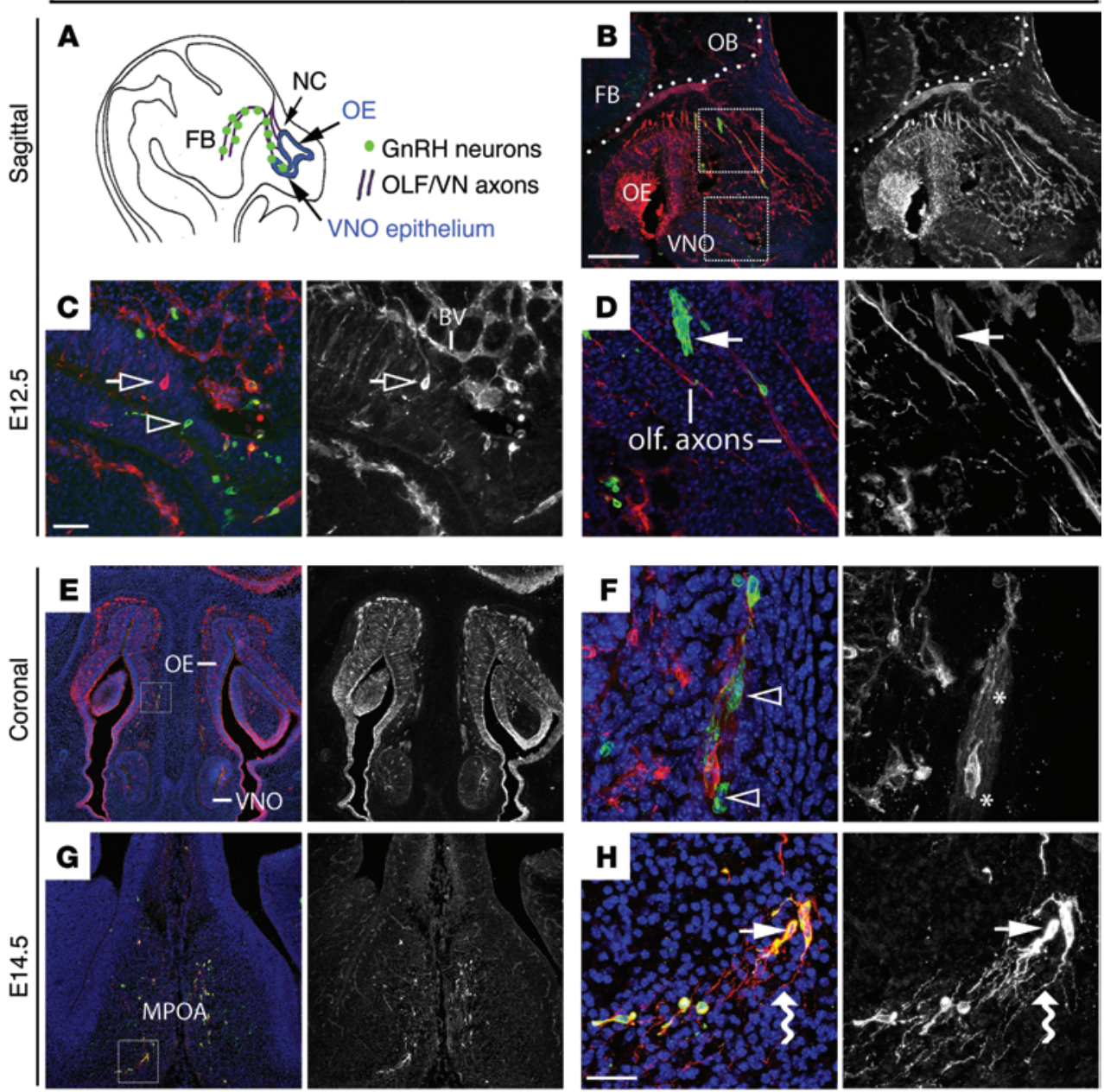

Figure 5. PLXND1 is expressed during GnRH neuron migration in the mouse. (A) Schematic representation of an embryonic mouse head to illustrate the migration of $\mathrm{GnRH}$ neurons (green dots) from the olfactory (OE) and VNO epithelia (blue) along OLF and VN axons (purple) through the nasal compartment (NC) into the forebrain (FB). (B-H) Sagittal and coronal sections of mouse heads at the indicated developmental stages, immunolabeled for $\mathrm{GnRH}$ and PLXND1; nuclei were counterstained with DAPI. Higher-magnification images of the areas indicated with dotted squares in $\mathbf{B}$ are shown in panels $\mathbf{C}$ and $\mathbf{D}$, and for $\mathbf{E}$ and $\mathbf{G}$ in $\mathbf{F}$ and $\mathbf{H}$, respectively. Adjacent to each panel in $\mathbf{B}-\mathbf{H}$, the corresponding single channels for PLXND1 are shown in grayscale. In C, clear arrows indicate PLXND1-positive, GnRH-negative cells, and clear arrowheads indicate examples of $\mathrm{GnRH}$-positive, PLXND1-negative cells in the OE. Solid arrows in $\mathbf{D}$ indicate GnRH neurons with low levels of PLXND1 in association with OLF axons with high levels of PLXND1. In F, clear arrowheads indicate PLXND1-negative GnRH neurons (their position is indicated with an asterisk). In $\mathbf{H}$, PLXND1-positive GnRH neurons in the MPOA and their neurites are indicated with straight and wavy arrows, respectively. Scale bars: $150 \mu \mathrm{m}$ (B, E, and $\mathbf{G}) ; 50 \mu \mathrm{m}$ (C, D, and F); $25 \mu \mathrm{m}(\mathbf{H})$.
The PLXND1 coreceptor KDR promotes SEMA3E-mediated GnRH neuron survival. To investigate why GN11 cells were not protected from cell death by SEMA3E like GT1-7 cells, we compared their expression of PLXND1 and the PLXND1 coreceptor KDR, also known as FLK1 or VEGFR2, because KDR was shown to be essential for PI3K activation during axonal growth of subicular neurons (11). Reverse transcriptase PCR (RT-PCR) analysis suggested that GN11 cells express PLXND1, although it was present at lower levels than in GT1-7 cells (Figure 3A). Interestingly, we detected KDR expression in GT1-7 (Figure 3A). In contrast, KDR was not expressed in GN11 cells (Figure 3A), which instead use NRP1 as a survival receptor (21). To test whether KDR was required for SEMA3E-mediated survival signaling in GT1-7 cells, we used a function-blocking antibody for $\operatorname{KDR}(10,21)$. While treatment of serum-starved cells with control IgG did not affect the survival of GT1-7 cells, the SEMA3E-mediated protection of GT1-7 cells was abrogated by anti-KDR (Figure 3, B-D; DMEM + IgG, 47\% $\pm 1.9 \% ; 10 \%$ FBS + IgG, $4.3 \% \pm 1.1 \% ; 10 \%$ FBS $+\alpha$ KDR, $8.8 \% \pm$ $1.7 \%$; SEMA3E + IgG, $14.1 \% \pm 1.3 \%$; SEMA3E + $\alpha$ KDR, $48.7 \% \pm$ $2.3 \%)$. Taken together, these observations suggest that SEMA3E signals through PLXND1 in a KDR-dependent fashion to promote PI3 kinase activation in maturing GnRH neurons.

SEMA3E, but not SEMA3ER619C, promotes GnRH neuron survival by activating PI3K signaling. To investigate whether the SEMA3E $E^{R 619 C}$ mutation impaired GnRH neuron survival by impairing AKT activation, we engineered expression constructs encoding human WT SEMA3E or SEMA3E ${ }^{\mathrm{R} 619 \mathrm{C}}$ protein and expressed them in COS cells to generate recombinant protein for tissue culture studies. Immunostaining and immunoblotting established that both proteins were expressed and secreted effectively (Figure 4, A and $B)$. Because we engineered recombinant SEMA3E as an alkaline phosphatase-conjugated ligand (AP-SEMA3E), we were also able to use it for binding assays. Binding of AP-SEMA3E to WT, but not to Plxnd1-null, mouse tissue established specificity of the AP-SEMA3E ligand (Figure 4C). Ligand-binding assays demonstrated that both WT SEMA3E and SEMA3E ${ }^{\mathrm{R} 619 \mathrm{C}}$ bound GT1-7 cells (Figure 4D). Even though mutant SEMA3E retained its ability to bind GT1-7 cells, it failed to protect them from death induced by serum starvation (Figure 4E; control, 38.3\% $\pm 0.9 \%$ vs. WT SEMA3E, $13.2 \% \pm 3.5 \%, P<0.001$; control vs. SEMA3E ${ }^{\mathrm{R} 619 \mathrm{C}}, 36.0 \% \pm 2.4 \%$; $P>0.5)$. Moreover, SEMA3E ${ }^{\mathrm{R} 619 \mathrm{C}}$ was ineffective in AKT activation in serum-starved GT1-7 cells (Figure 4F). Taken together, these observations suggest that SEMA3E-mediated survival signaling in maturing GnRH neurons is compromised by the SEMA3E p.R619C mutation.

PLXND1 expression during mouse GnRH neuron development. The tissue culture studies above suggested that SEMA3E signaling is essential to promote the survival of maturing GnRH neurons, i.e., GnRH neurons at late stages of embryonic development. To 
A

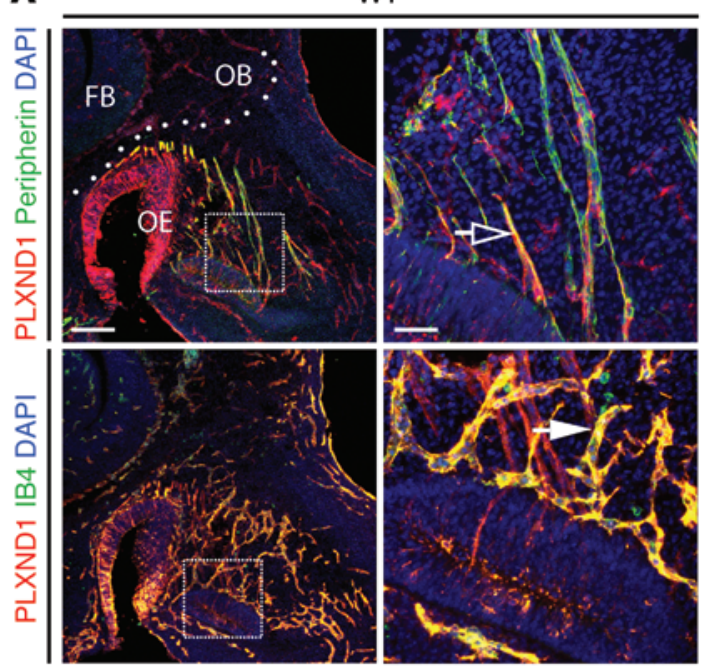

C

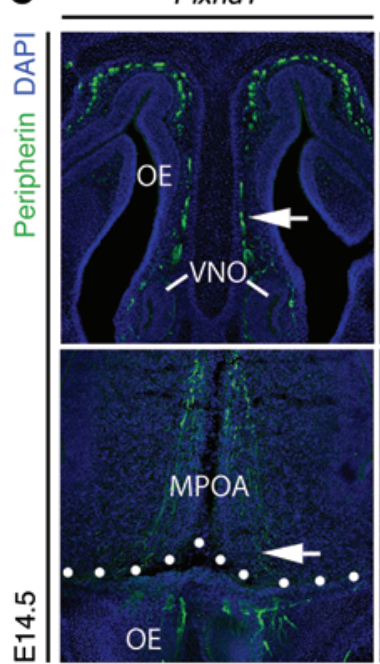

Plxnd1--

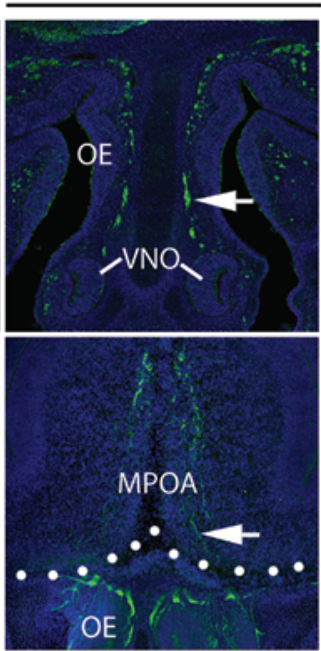

B

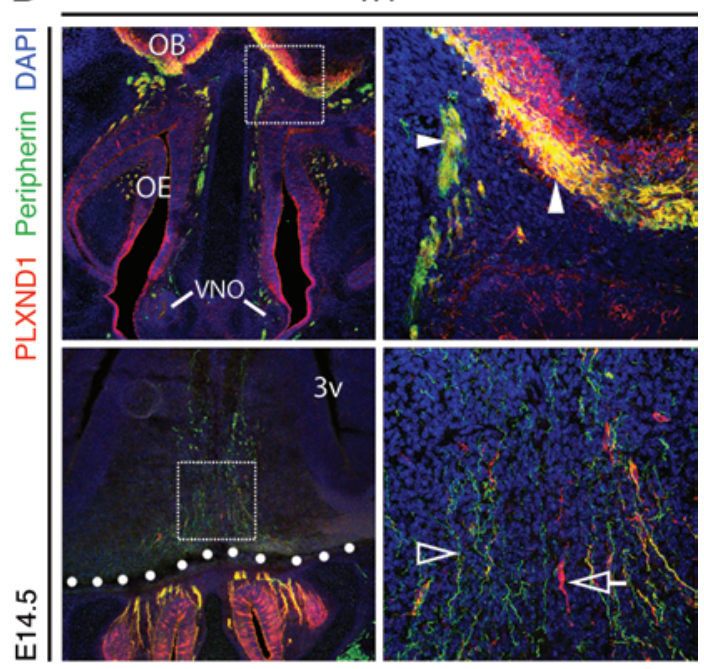

D

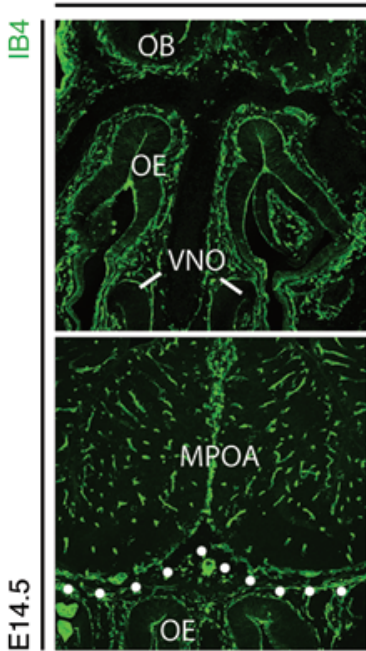

Plxnd1 1 -

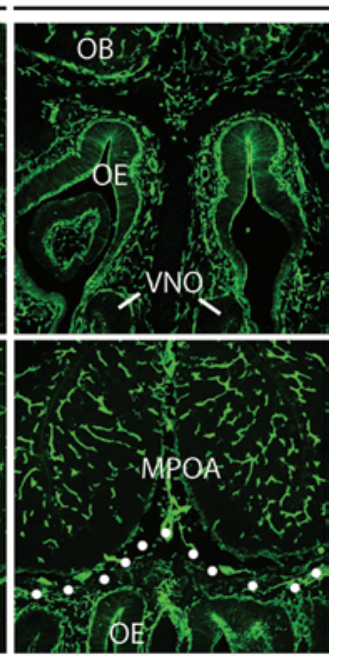

Figure 6. PLXND1 is dispensable for axonal and vascular patterning in the embryonic mouse nose. (A) PLXND1 expression in E12.5 mouse nose. Sagittal sections of E12.5 WT heads, immunolabeled for PLXND1 and the OLF/VN axonal marker peripherin (top panels) or the vascular marker IB4 (bottom panels); sections were counterstained with DAPI. Higher-magnification images of the areas indicated with dotted squares are shown in the adjacent panels. The clear arrow indicates a PLXND1-positive axon and the solid arrow a PLXND1-positive blood vessel. (B) PLXND1 expression in E14.5 mouse nose and brain. Coronal sections of E14.5 WT nose (top panels) and MPOA (bottom panels), immunolabeled for PLXND1 and peripherin; sections were counterstained with DAPI. Higher-magnification images of the areas indicated by dotted boxes are shown in the adjacent panels. PLXND1 expression in axons in the nose and OLF bulbs $(\mathrm{OB})$ is indicated with arrowheads. Note PLXND1 expression by GnRH neurons in the MPOA (clear arrow), but not by the caudal branch of the VN nerve (clear arrowhead). 3v, third ventricle. (C and D) Normal nasal axon and nasal/brain blood vessel patterning in PIxnd1-null mutants. Coronal sections of E14.5 mouse heads of the indicated genotypes at the level of the nose (top panels) and MPOA (bottom panels) were immunolabeled for peripherin and counterstained with DAPI (C) or labeled with the blood vessel marker IB4 (D). Dotted lines indicate forebrain boundaries. Scale bars: $150 \mu \mathrm{m}$ (A-D), $50 \mu \mathrm{m}$ (higher-magnification images of boxed areas in $\mathbf{A}$ and $\mathbf{B}$ ).

determine the expression pattern of PLXND1 and its functional requirement for $\mathrm{GnRH}$ neuron development, we used the mouse as a model organism. Most mouse GnRH neurons arise between E10.5 and E12.5 in the nasal placode that also gives rise to the olfactory epithelium (OE) and vomeronasal organ (VNO) (4). Newly born GnRH neurons leave the placode by migrating along olfactory (OLF) and vomeronasal (VN) axons toward the forebrain, which they enter by following along the caudal branch of the VN/terminal nerve (Figure 5A). Immunofluorescence labeling of sagittal sections identified abundant PLXND1 expression in the
E12.5 head (Figure 5B). In particular, PLXND1 localized to nasal axons and blood vessels as well as to the OE and VNO epithelia (Figure 5C). However, most GnRH-positive cells (corresponding to GnRH neurons) within the $\mathrm{OE}$ and VNO or elsewhere in the nose appeared only faintly positive or negative for PLXND1 (Figure 5, C and D). Immunofluorescence labeling of coronal sections through E14.5 heads showed a similar pattern of PLXND1 expression in the OE and VNO, but absent or faint expression on GnRH neurons in the nose (Figure 5, E and F). Although only weakly positive for PLXND1 while migrating in the nose, GnRH neurons 
A $\mathrm{E} 14.5$

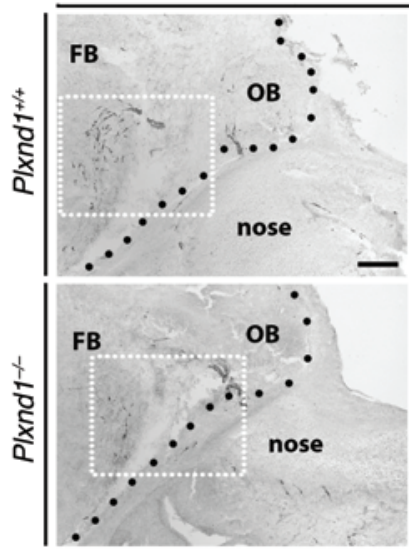

GnRH

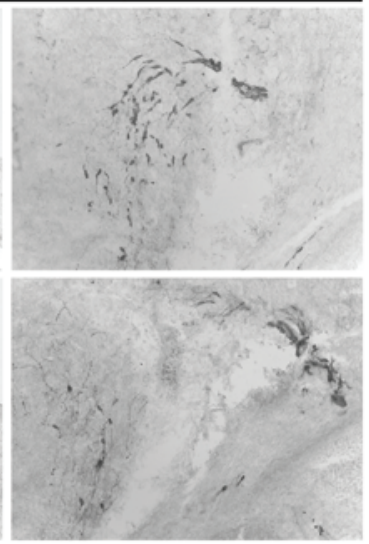

B

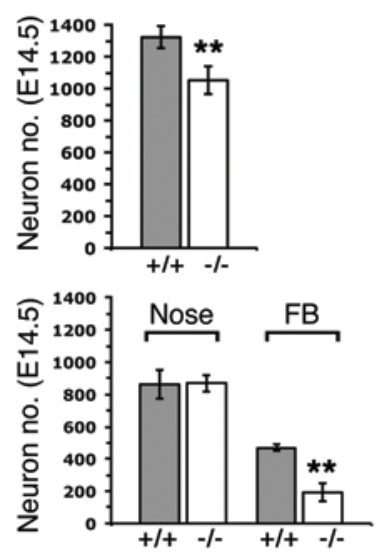

C $\mathrm{E} 17.5$

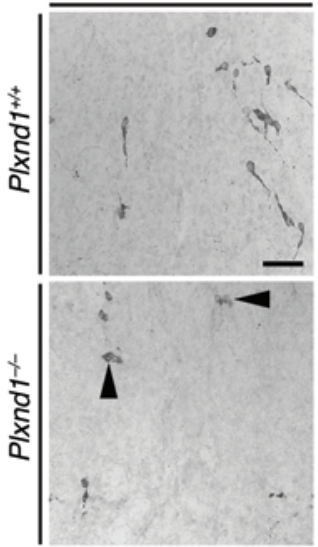

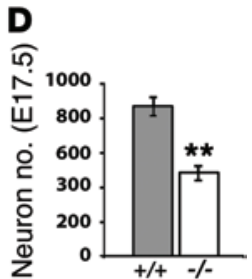

F

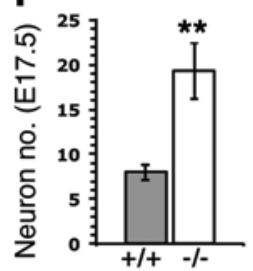

E $E 14.5$

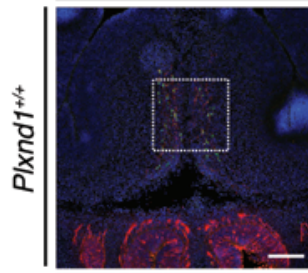

PLXND1 GnRH DAPI
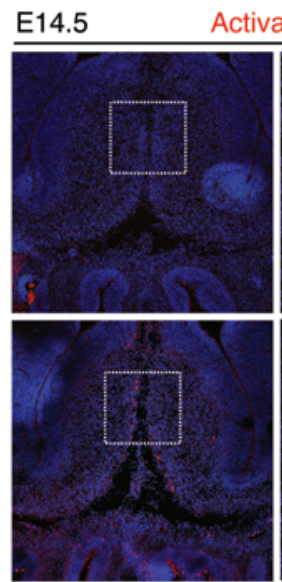
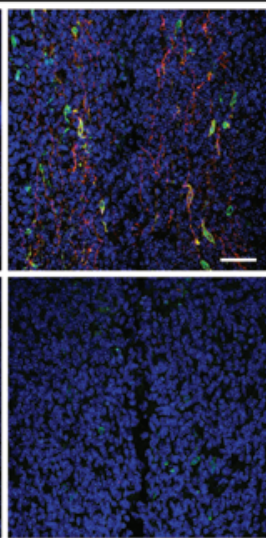

Activated caspase-3 DAPI

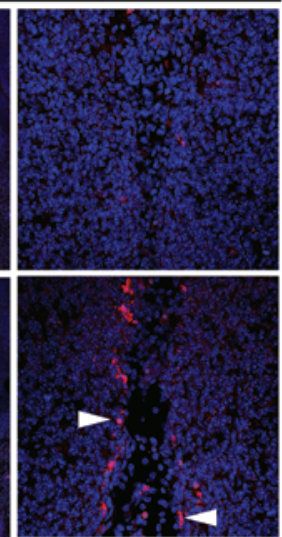

G E14.5
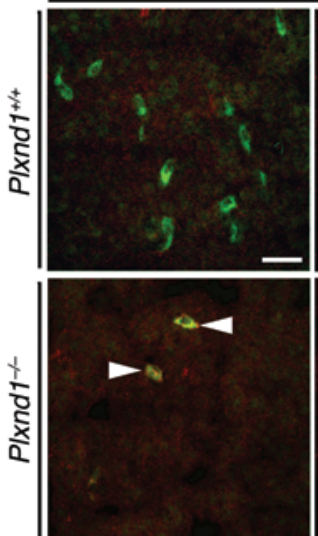

Gnrh Activated caspase-3

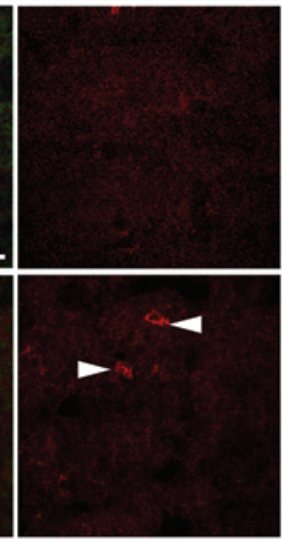

Figure 7. Reduced GnRH neuron numbers and increased apoptosis correlate in the PIxnd1-null MPOA. (A and B) PLXND1 loss caused GnRH neuron loss after the forebrain. Sagittal sections of E14.5 mouse heads were immunolabeled for $\mathrm{GnRH}$, revealing fewer GnRH neurons in mutant compared with WT heads (A). Dotted lines indicate the boundary between the nose and forebrain. White dotted squares indicate regions shown at higher magnification in the adjacent panels. (B) Quantitation of GnRH neuron distribution shows that $\mathrm{GnRH}$ neuron loss was only significant in the mutant forebrain, but not the nose ( $n=3$ each; ${ }^{* *} P<0.01$, Student's $t$ test). (C and D) PLXND1 loss caused GnRH neuron loss in the MPOA. (C) Coronal sections of E17.5 MPOA were immunolabeled for $\mathrm{GnRH}$, revealing fewer $\mathrm{GnRH}$ neurons in the mutant mice than in the WT mice. (D) Quantitation confirmed a significant reduction of GnRH neuron numbers in PLXND1-deficient compared with WT MPOA ( $n=3$ each; ${ }^{* *} P<0.01$, Student's $t$ test). (E and F) PLXND1 loss increased apoptosis in the MPOA. (E) Adjacent coronal sections of E14.5 MPOA were immunolabeled for GnRH together with PLXND1 or activated caspase-3, revealing apoptotic cells in areas containing GnRH neurons (indicated with solid arrowheads). Higher-magnification images of areas indicated with dotted squares are shown in the adjacent panels. (F) Quantitation shows increased cell death in the MPOA of Plxnd1-null versus WT mice ( $n=3$ each; ${ }^{* *} P<0.01$, Student's $t$ test). (C) PLXND1 loss caused GnRH neuron apoptosis. Gnrh ISH of coronal sections from E14.5 MPOA followed by immunolabeling for activated caspase-3 revealed apoptotic GnRH neurons (indicated with solid arrowheads). Scale bars: $150 \mu \mathrm{m}$ (A and E), $50 \mu \mathrm{m}$ (C and higher-magnification images of boxed areas in E), $45 \mu \mathrm{m}$ (G).

that had entered the forebrain began to express PLXND1; moreover, GnRH neurons in the medial preoptic area (MPOA), where most hypothalamic GnRH neurons eventually reside, expressed PLXND1 strongly on both their cell bodies and axons (Figure 5, G and $\mathrm{H} ; 81.6 \% \pm 7.9 \%$ of $\mathrm{GnRH}^{+}$cells were also PLXND1 ${ }^{+}$). These observations are consistent with a requirement for SEMA3E signaling through PLXND1 in developing GnRH neurons after they have entered the brain, consistent with the in vitro analysis, which suggested a role for SEMA3E in GnRH neurons at an advanced stage of differentiation. In addition, the expression pattern may indicate possible roles for PLXND1 in vascular and OLF development. We therefore examined whether disrupted vascular patterning, abnormal OLF axon guidance, and/or cell-autonomous defects in GnRH neurons might contribute to the loss of GnRH neurons in PLXND1-null mice.
PLXND1-null mutants show normal axonal and blood vessel patterning in the nose and forebrain. Mice lacking neuropilin signaling show ectopic GnRH neuron migration along mispatterned axons in the nose and an ensuing accumulation of ectopic GnRH neurons outside the brain (5). Because PLXND1 was present on nasal axons at E12.5 (Figure 6A) and E14.5 (Figure 6B), we examined whether nasal axon tracts were also defective in Plxnd1-null mice, but found no obvious abnormalities (Figure 6C). In addition, the caudal branch of the VN/terminal nerve that guides GnRH neurons into the forebrain appeared PLXND1 negative (Figure 6B) and projected normally in Plxnd1-null mice (Figure 6C), excluding a role for PLXND1 in directing GnRH neuron migration into the brain. Because PLXND1 was previously implicated in trunk and retinal vascular patterning $(9,10)$ and is expressed by blood vessels in both the nose and forebrain 

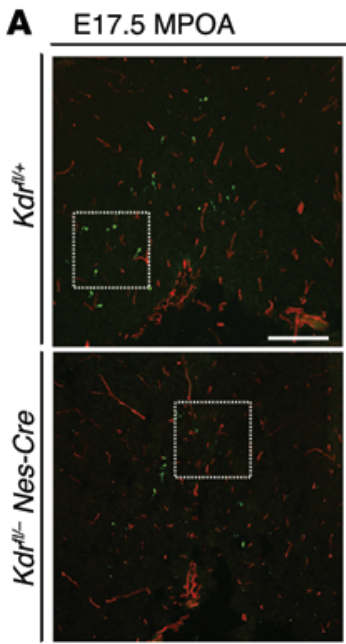
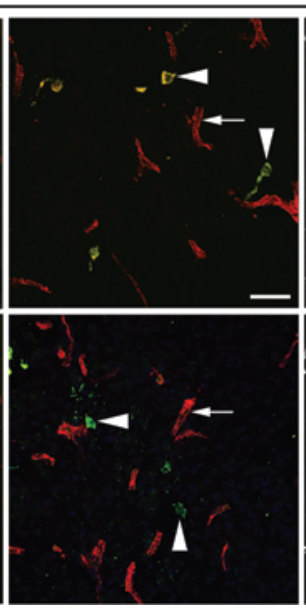

GnRH KDR
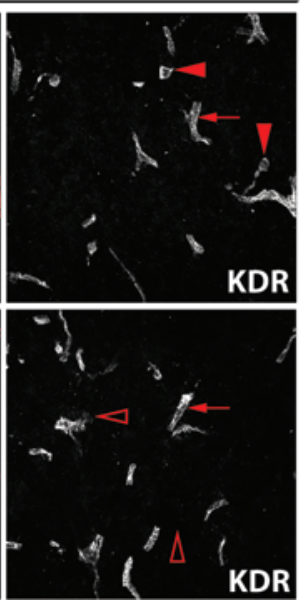

B
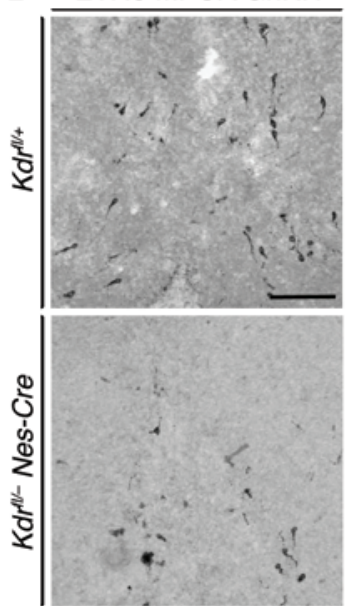

C

$\square K d r^{n / 4+}$

$\square K d r^{n /-}$ Nes-Cre

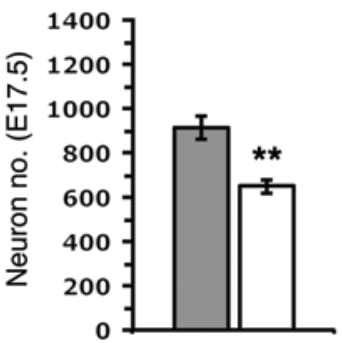

Figure 8. KDR deficiency decreases GnRH neuron numbers in the brain. (A) GnRH neurons expressed KDR in the brain. Coronal sections of E17.5 mouse MPOA of the indicated genotypes were immunolabeled for GnRH and KDR. Higher-magnification images of the areas indicated by dotted boxes and corresponding single channels for KDR are shown adjacent to each panel. White arrowheads indicate examples of GnRH-positive cells; red arrowheads indicate KDR staining in the same cells in the single-channel images. Clear red arrowheads indicate GnRH-positive cells with KDR knockdown. Small white and red arrows indicate KDR-positive blood vessels. (B and $\mathbf{C}$ ) Reduced number of $\mathrm{GnRH}$ neurons in the brain of mice lacking KDR in neurons. Coronal sections of E17.5 mouse MPOA from the indicated genotypes were immunolabeled for $\mathrm{GnRH}(\mathbf{B})$ and the number of $\mathrm{GnRH}$ neurons quantitated (C) ( $n=3$ each; ${ }^{* *} P<0.01$, Student's $t$ test). Scale bars: $150 \mu \mathrm{m}$ and $50 \mu \mathrm{m}$ (low- and high-magnification images in A, respectively), $50 \mu \mathrm{m}$ (B).

during GnRH neuron migration (Figure 5C and Figure 6A), we next examined whether PLXND1-null mutants have insufficient tissue vascularization that could indirectly affect $\mathrm{GnRH}$ neuron development. However, fluorescence staining of E14.5 head sections with the vascular marker IB4 did not identify any obvious differences in blood vessel organization in the nose or brain between mutants and their littermate controls (Figure 6D). Taken together, these data are consistent with a cell-autonomous role for PLXND1 signaling in GnRH neurons, rather than indirect effects of PLXND1-dependent OLF/VN nerves or blood vessels on GnRH neuron development.

PLXND1 loss impairs GnRH neuron survival in the developing mouse brain. Because we had observed that PLXND1 promoted SEMA3E-mediated survival signaling in a GnRH neuron tissue culture model and found high expression of PLXND1 in forebrain GnRH neurons in situ, we next asked whether Plxnd1-null mice (9) lacked GnRH neurons. IHC for GnRH of sagittal sections followed by quantitation of all GnRH-positive cells in each head demonstrated a significant reduction in the number of $\mathrm{GnRH}$ neurons in mutants compared with WT mouse heads at E14.5 (Figure 7, A and B; $P l x n d 1^{+/+}, 1,322 \pm 34$ vs. $\left.P l x n d 1^{-/}, 1,051 \pm 43 ; P<0.01\right)$. Because $\mathrm{GnRH}$ neurons at E14.5 reside in both the nose and forebrain, we next determined the relative amount of neurons in both compartments. Whereas the number of GnRH-positive cells was similar in the nose of both genotypes, their numbers were significantly reduced in the forebrain of mutants compared with WT (Figure 7B; nose: $P l x n d 1^{+/+}, 861 \pm 13$ vs. $P l x n d 1^{-/}, 867 \pm 31, P>0.05$; forebrain: $P l x n d 1^{+/+}, 469 \pm 51$ vs. $\left.P l x n d 1^{-/-}, 191 \pm 29, P<0.01\right)$.

To exclude that the reduced number of GnRH neurons in the mutant brain at E14.5 was not caused by a developmental delay, we examined the MPOA of Plxnd1-null mutants and their littermates during late embryogenesis. While many GnRH neurons were present in the WT MPOA at E17.5, GnRH neurons were sparse in littermate mutants, and residual neurons appeared collapsed (Figure 7, C and D; Plxnd1 $1^{+/}, 933 \pm 21$ vs. Plxnd1 $1^{-/}, 486 \pm$ 26, $P<0.001$; heterozygous mice had a normal number of $\mathrm{GnRH}$ neurons: Plxnd1 ${ }^{+/-}, 979 \pm 18 ; n=3$ ). Together, these findings suggest that PLXND1 is specifically required to maintain a normal number of GnRH neurons after they have entered the brain. These results also agree with the observed PLXND1 upregulation in GnRH neurons in the brain relative to the nose and the responsiveness of mature GT1-7, but not immature GN11, cells to SEMA3E-induced survival signaling in vitro.

We next examined whether PLXND1 is required for the survival of GnRH neurons in the developing brain. For this analysis, we labeled adjacent coronal sections through the MPOA, where most GnRH neurons reside after E14.5, with antibodies against GnRH and PLXND1 or activated caspase-3, a marker for cells committed to undergo apoptosis (Figure 7E). We identified many GnRH neurons, but few apoptotic cells, in the WT MPOA (Figure 7E, upper panels); in contrast, GnRH neurons were sparse and apoptotic cells prominent in the mutant MPOA (Figure 7E, right lower 2 panels). This experiment confirmed the specificity of the PLXND1 antibody used, as it did not label mutant tissue (Figure 7E, left lower 2 panels). Quantitation confirmed a significant increase in the number of apoptotic cells in the mutant MPOA at E14.5 (Plxnd1 ${ }^{+/+}, 8 \pm 0.5$ vs. Plxnd1 $1^{-/}, 21 \pm 1.8, P<0.01$ ) (Figure $7 F$ ). Because we could not identify suitably compatible antibodies to detect GnRH, PLXND1, and activated caspase- 3 by triple immunolabeling, we instead combined Gnrh ISH with immunostaining for activated caspase-3 to demonstrate directly that GnRH neurons undergo apoptosis when they have reached the MPOA (arrowheads, Figure 7G). Finally, we combined immunofluorescence for GnRH with TUNEL staining (28) at E14.5 to identify GnRH neurons in the terminal stages of apoptosis (Supplemental Figure 1; supplemental material available online with this article; doi:10.1172/JCI78448DS1). Together, these 
A GnRH PLXND1 DAPI
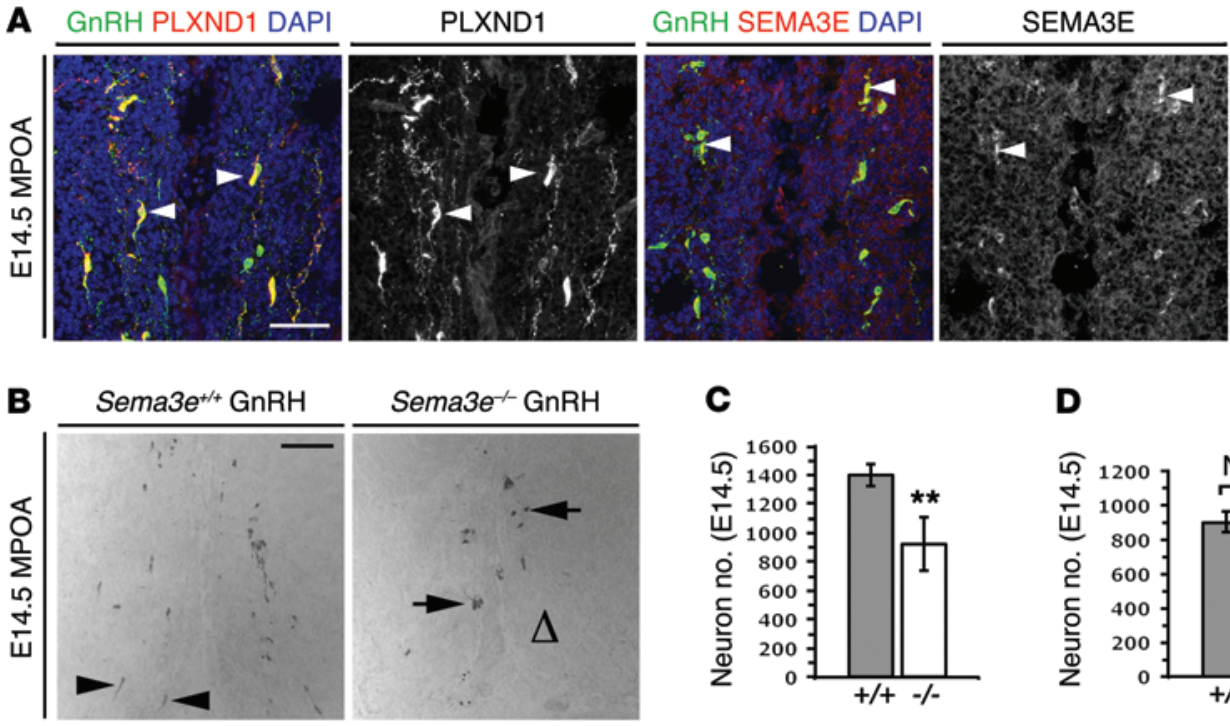

Sema3e $e^{--} \mathrm{GnRH}$

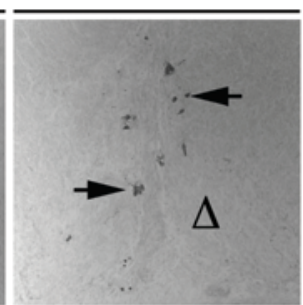

C

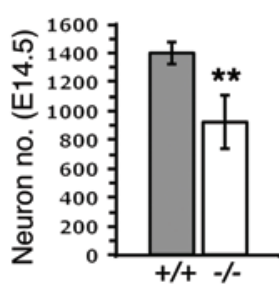

D

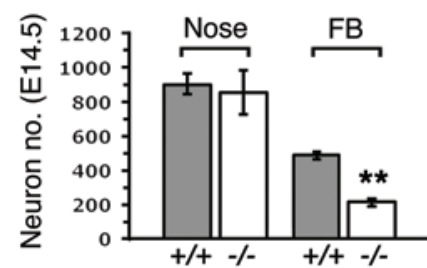

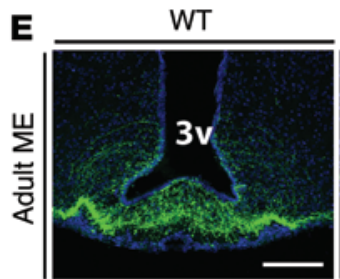
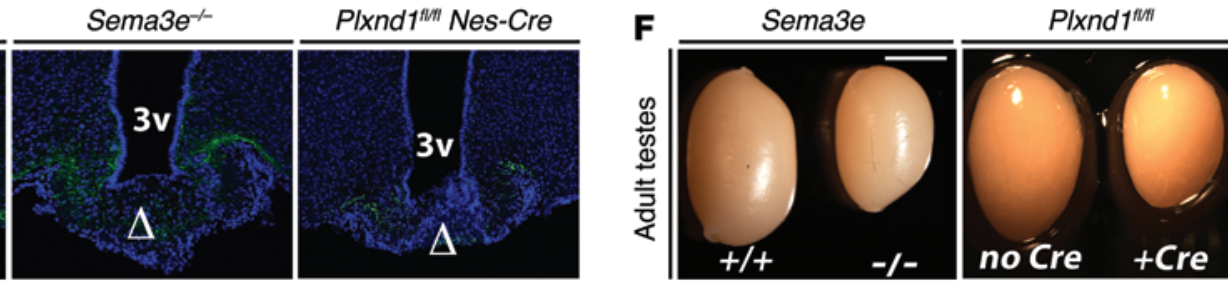

Figure 9. SEMA3E deficiency reduces GnRH neuron numbers, projection to the ME, and testes size. (A) SEMA3E in the MPOA. Adjacent coronal sections of E14.5 mouse MPOA, immunolabeled for GnRH and PLXND1 or SEMA3E; the SEMA3A single channel is shown adjacent to the triple label. Arrowheads indicate examples of PLXND1- and GnRH-positive neurons stained for SEMA3E, consistent with SEMA3E binding to PLXND1. (B-D) SEMA3E loss reduced GnRH neuron numbers. (B) Coronal sections of E14.5 MPOA were immunolabeled to identify GnRH neurons; $\Delta$ denotes neuron deficiency in mutants compared with controls; arrowheads indicate examples of GnRH neurons with a normal, elongated morphology; black arrows indicate rounded GnRH neurons in the mutants. (C) Quantitation revealed a significant reduction in $\mathrm{GnRH}$ neuron numbers in Sema3e mutants compared with WT littermates. $n=3$; ${ }^{* *} P<0.01$ by Student's $t$ test. (D) Quantitation revealed a significant reduction of GnRH neuron numbers in Semaze mutant relative to WT forebrain, but normal numbers in the nose. $n=3$; ${ }^{* *} P<0.01$ by Student's $t$ test. (E) Loss of SEMA3E or neural PLXND1 reduced GnRH neuron projections to the ME. Coronal sections of adult hypothalamus were immunostained to identify GnRH-positive axons (green) projecting to the ME (counterstained with DAPI, blue); innervation was poor in mutants compared with that in WT (indicated by $\Delta$ ). (F) Loss of SEMA3E or neural PLXND1 reduced testes size. Testes pairs from adult littermate males were photographed side by side to demonstrate the reduced size of testes in SEMA3E-null and neuron-specific PLXND1 mutants. Scale bars: $25 \mu \mathrm{m}$ (A); $50 \mu \mathrm{m}$ (B); $50 \mu \mathrm{m}$ (E); $300 \mu \mathrm{m}$ (F).

experiments show that loss of PLXND1 signaling compromises GnRH neuron survival in the developing brain.

Loss of the PLXND1 coreceptor KDR decreases GnRH neuron numbers in the brain. Because our in vitro experiments suggested that SEMA3E-mediated survival signaling through PLXND1 was dependent on the PLXND1 coreceptor FLK1, we examined whether GnRH neurons in the MPOA expressed KDR and whether KDR was required for their survival. We observed that GnRH neurons in the E17.5 MPOA of control mice expressed KDR (Figure 8A, top panels). In contrast, $\mathrm{Kdr} \mathrm{r}^{\mathrm{tl}-} \mathrm{Nes}$-Cre-mutant mice lacking KDR in the neural lineage (11) showed only faint expression of KDR in GnRH neurons (Figure 8A). Importantly, blood vessels retained KDR immunoreactivity in the mutants, demonstrating specificity of the neural $\mathrm{KO}$ (Figure 8A, bottom panels). This experiment also showed that fewer GnRH neurons were present in the E17.5 MPOA of mutants compared with that seen in controls (Figure 8A, bottom panels). We therefore immunostained sections through the entire MPOA of E17.5 mutants and controls and then quantified the number of GnRH neurons (Figure 8, B and C). This analysis demon- strated a significantly reduced number of GnRH neurons in $\mathrm{Kdr} r^{\mathrm{fl} /-}$ Nes-Cre mutants compared with that in control littermates (Figure $8 \mathrm{C} ; K_{d r^{f l /+}}, 910 \pm 30$ vs. Kdrfl- Nes-Cre, $\left.650 \pm 18 ; P<0.01\right)$. These observations agree with the finding that GT1-7 cells require KDR for SEMA3E-mediated survival signaling via PLXND1 in vitro.

Loss of the PLXND1 ligand SEMA3E decreases GnRH neuron numbers in the brain. Consistent with a role for SEMA3E survival signaling in GnRH neurons in the MPOA, SEMA3E localized to PLXND1-positive GnRH neurons and was present in their environment (Figure 9A). IHC staining, followed by counting of all GnRH-positive cells in each head, demonstrated a significant reduction in the number of GnRH neurons in Sema3e-null mutants compared with WT littermates at E14.5 (Figure 9, B and C; Sema3e H $^{++}, 1,400 \pm 47$ vs. Sema3e $e^{-/}, 922 \pm 106$; $\left.P<0.01\right)$. Moreover, residual neurons in the MPOA at E14.5 appeared collapsed (Figure 9B), and neuron loss was pronounced in the forebrain but not the nose (Figure 9D; nose: Sema3e $^{+/+}, 905 \pm 36$ vs. Sema3e $e^{-/-}, 857 \pm 75, P>0.05$; forebrain: Sema3e $e^{+/+}, 486 \pm 12$ vs. Sema3e $\left.e^{-/}, 214 \pm 14, P<0.01\right)$. The phenotypic similarity of mice lacking SEMA3E with that of mice 
A

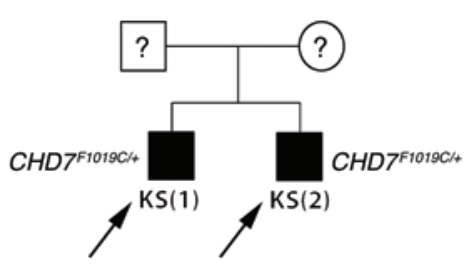

C 1015-IHGPFLVIAPLSTIPN-1030 HUMAN 1005-IHGPFLVIAPLSTIPN-1020 MOUSE 1004-IHGPFLVIAPLSTIPN-1019 RAT 1014-IHGPFLVIAPLSTIPN-1031 DOG 1014-IHGPFLVIAPLSTIPN-1031 DOG
965 -IHGPFLVIAPLSTIPN- 980 CATTLE 1016-IHGPFLVIAPLSTIPN- 1031 CHICK 1059-IHGPFLVIAPLSTIPN-1075 ZEBRAFISH
B

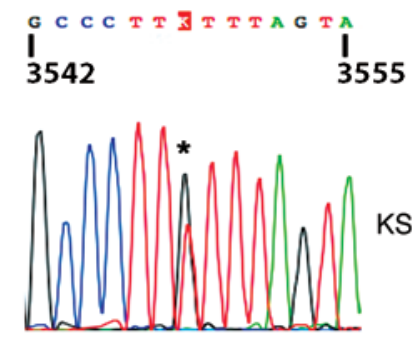

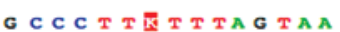

(1)

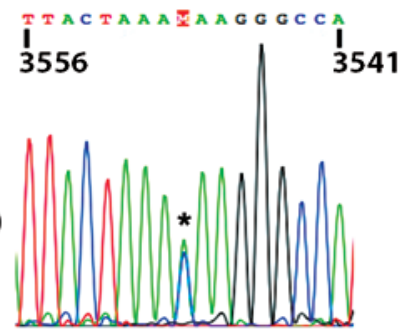

KS (2)

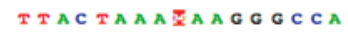

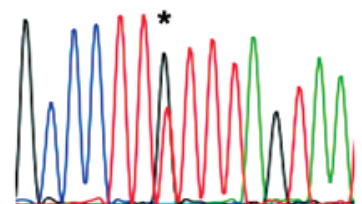
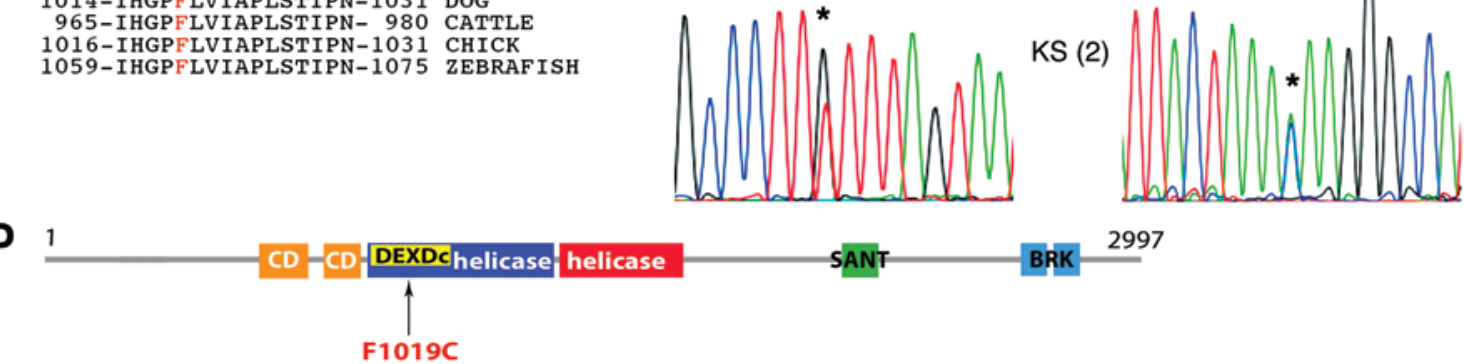

E

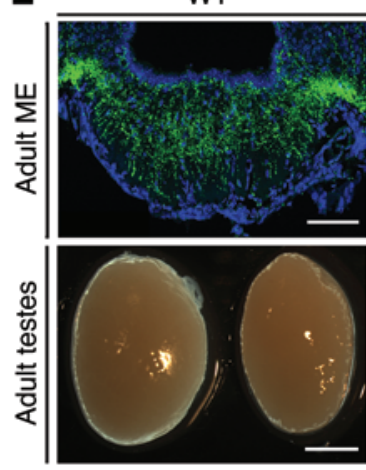

Chd7 $7^{\text {t- }}$

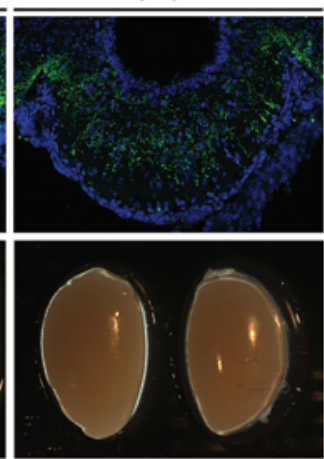

Plxnd1+/- Chd7 $7^{\text {+/ }}$

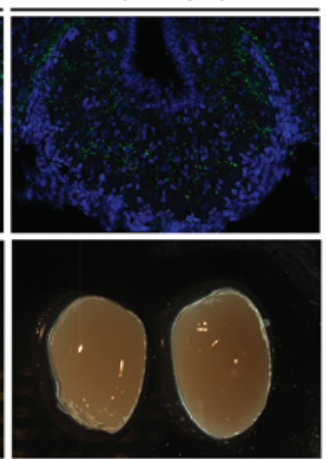

$\mathbf{F}$

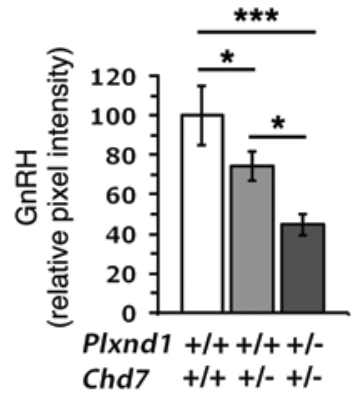

Figure 10. Genetic interaction of SEMA3E and CHD7 in KS. (A) Pedigree of the 2 brothers affected by KS. A heterozygous p.F1019C mutation in CHD7 is present in the brothers with the p.R619C mutation in SEMA3E. Circle denotes female; square denotes male; black square denotes affected male; arrows indicate the probands. (B) Identification of the CHD7 mutation. Sequence chromatograms revealed a heterozygous nucleotide substitution (T>C, asterisks) in exon 12 of CHD7. (C) The CHD7 mutation affects an evolutionarily conserved amino acid residue. Alignment of partial protein sequences of $C H D 7$ orthologs shows that F1019 (red) is evolutionarily conserved. (D) CHD7 domain structure. The position of the mutation is indicated. CD, chromodomain; DEXDc, deadlike helicase domain with ATP-binding pocket; SANT, histone interaction domain; BRK, Brahma and Kismet domain. (E and F) Genetic interaction of Chd7 and PIxnd1. Coronal sections of hypothalamus from adult littermate mice of the indicated genotypes, immunostained to identify GnRH-positive axons at the $\mathrm{ME}(\mathbf{E})$, and the quantitation of $\mathrm{GnRH}$ pixel intensity in the immunostained sections (F), show more severely reduced ME innervation in compound compared with single mutants. $n=3$ each; ${ }^{*} P<0.05$ and ${ }^{* * *} P<0.001$ by 1 -way ANOVA. The testes size (E) was correspondingly reduced. Scale bars: $25 \mu \mathrm{m}$ (ME in E), $400 \mu \mathrm{m}$ (testes in $\mathbf{E}$ ).

lacking PLXND1 or KDR agrees with the idea that SEMA3E serves as a ligand for a PLXND1/KDR coreceptor complex to promote the survival of GnRH neurons in the developing brain.

Loss of SEMA3E or neuronal PLXND1 decreases innervation of the adult median eminence by GnRH neurons and the size of adult testes. In adults, GnRH neurons project to the median eminence (ME), where they release GnRH into the portal blood vessels of the pituitary gland. We observed a markedly reduced number of GnRH-positive neurites at the ME in adult Sema3e-null compared with WT males (Figure 9E). We also examined ME innervation in Plxnd1 mutants. For this experiment, we created neuron-specific PLXND1-null mice (Plxnd $1^{f l / f l}$ Nes-Cre; refs. 29, 30) to circumvent the neonatal lethality of full Plxnd1-null mice, which is caused by endothelial cell defects in the cardiac outflow tract $(9,31)$. Similar to Sema3e-null mutants, the ME of Plxnd1 $1^{f / f l}$ Nes-Cre adult males was poorly innervated by GnRH-positive axons (Figure 9E; GnRH pixel intensity reduction in ME neurites in mutants relative to that seen in WT littermates: Sema3e $e^{-/}$, $36 \% \pm 0.8 \%$; Plxnd1 $1^{f l f l}$ Nes-Cre, $\left.49 \% \pm 1.6 \%\right)$. This experiment also provided additional evidence for a cell-autonomous function of PLXND1 rather than roles for PLXND1-dependent vasculature in GnRH neuron survival.

Impaired GnRH neuron innervation of the ME is predicted to reduce gonadotropin secretion from the pituitary and thereby affect normal sexual development. Consistent with this idea, and in agreement with the phenotype of patients carrying the 
$S E M A 3 E^{R 619 C}$ mutation, the testes of adult male mice lacking SEMA3E or neuronal PLXND1 were smaller than those of WT littermates (Figure 9F). On the basis of these findings in genetic mouse models and combined with the identification of a SEMA3E mutation in 2 brothers with KS and our tissue culture studies with recombinant SEMA3E, we conclude that loss of SEMA3E, either through genetic deletion or mutation, contributes to the etiopathogenesis of inherited GnRH deficiency, including KS.

A heterozygous missense mutation in CHD7 accompanies the R619C mutation in SEMA3E in the KS brothers. While the mouse mutants we have examined here were bred to homozygosity to define a role for SEMA3E in GnRH neuron development, the R619C mutation in $S E M A 3 E$ we identified in the $2 \mathrm{KS}$ brothers was present in a heterozygous pattern. This finding agrees with the commonly observed oligogenic inheritance pattern of KS in the human population, which is exemplified by various combinations of previously identified heterozygous mutations in the SEMA3A, FGFR1, NELF, KAL1, PROKR2, and PROK2 genes (7, 32-36). We therefore examined the exome sequence of both KS brothers for mutations in all other previously identified KS genes, but found no such mutations except the same missense mutation in the gene encoding the chromodomain helicase DNA-binding protein 7 (CHD7) in both brothers (Figure 10, $A$ and $B$ ). This nucleotide substitution replaced the codon for phenylalanine with a codon for cysteine (p.F1019C) and was not present in any other exome in the 1000 Genomes and ESP databases.

CHD7 is a chromatin remodeler that modulates gene transcription during the development of multiple organ systems (37, 38), and CHD7 mutations are the main cause of CHARGE syndrome (coloboma, heart defect, choanal atresia, retardation of growth and/or development, genital hypoplasia, ear anomalies) (39). However, while CHARGE patients commonly carry severe truncating mutations in $\mathrm{CHD7}$, missense mutations in $\mathrm{CHD7}$ are more prevalent in KS patients (40). In agreement with these earlier observations, the $C H D 7$ mutation we identified in the brothers with $\mathrm{KS}$ is a missense mutation that was predicted to be possibly damaging, deleterious, or disease causing by PolyPhen-2, SIFT, and MutationTaster bioinformatic tools, respectively (Table 1). Thus, the F1019C mutation affects an amino acid residue that is highly conserved between mammals and lower vertebrate species (Figure 10C) and is positioned on the DEAD-like helicase domain (DEXDC) that is essential for ATP binding (Figure 10D).

In summary, the coexistence of the same heterozygous missense mutation in CHD7 mutations in the 2 brothers with a 2-year age difference suggests that both mutations were inherited from their fertile parents and is consistent with the idea that CHD7 functions cooperatively with $S E M A 3 E$ in a genetic pathway essential for GnRH neuron development.

Genetic interaction of PLXND1 and CHD7 in the GnRH neuron system. Because the heterozygous loss of PLXND1 did not impair GnRH neuron development (see above), we were able to examine the genetic interaction of PLXND1 and CHD7. Thus, we mated $\mathrm{Plxnd1}^{+/}$females with $\mathrm{Chd7}^{+/}$males (41) and examined the innervation of the $\mathrm{ME}$ in single and compound heterozygous mice. We found that fewer GnRH-positive neuron fibers projected to the ME in the hypothalamus of their $\mathrm{Chd7}^{+/-}$male offspring compared with that seen in their WT littermates (Figure 10E, top panels), consistent with previous observations (42). Importantly, the finding that
$C h d 7^{+/-}$males were able to sire offspring suggested that the heterozygous loss of CHD7 function affects the GnRH neuron system at a subclinical level. Strikingly, compound heterozygous $\mathrm{Plxnd1}^{+-}$ $C h d 7^{+/-}$males showed a more pronounced reduction in the intensity

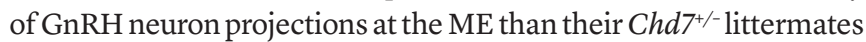
with normal PLXND1 levels (Figure 10E, top panels). Similar results were also obtained for female mice (Figure 10F; reduced GnRH pixel intensity at the mutant ME relative to the WT female ME at P14: $\mathrm{Chd7}^{+/-} \mathrm{Plxnd1}^{+/+}, 25.5 \% \pm 3.1 \%, \mathrm{P}<0.05 ; \mathrm{CHD}^{+/-} \mathrm{Plxnd1}^{+/-}$, $55.2 \% \pm 2.5 \%, P<0.001)$. Consistent with reduced $\mathrm{ME}$ innervation and also with the phenotype of the 2 brothers carrying the SEMA3E and $\mathrm{CHD} 7$ mutations, the testes of adult $P l x n d 1^{+-} \mathrm{Chd7}^{+/-}$compound heterozygous males were smaller than those of WT and $C h d 7^{+/-}$littermate males (Figure 10E, bottom panels). Together, these observations suggest that CHD7 and PLXND1 interact genetically to enable formation of the GnRH neuron system.

\section{Discussion}

$\mathrm{KS}$ is a rare genetic condition that impairs puberty and human reproduction and can be treated if diagnosed early. Here, we have combined exome sequencing of patients with bioinformatics analysis, tissue culture experiments, and animal models to identify $S E M A 3 E$ as a novel KS gene that controls GnRH neuron development. The heterozygous nature of the SEMA3E mutation we identified agrees with the current model of oligogenic inheritance of various combinations of heterozygous mutations in the SEMA3A, FGFR1, NELF, KAL1, PROKR2, and PROK2 genes (7, 32-36). In agreement with this finding, the heterozygous SEMA3E mutation was accompanied by a heterozygous missense mutation in CHD7 that was not present in any other exome in the 1000 Genomes and ESP databases. Importantly, the presence of the same SEMA3E and $C H D 7$ mutations in brothers with a 2-year age difference suggests that both mutations were inherited. However, lack of access to parental DNA prevented our ability to investigate whether the SEMA3E mutation was inherited through the paternal or maternal lineage, and whether the CHD7 mutation was inherited from the other parent, as may be predicted from the fertility of the parents, but infertility of their children, who combined both mutations. Supporting the idea of oligogenic inheritance and an interaction of the genes encoding SEMA3 family members and CHD7, two KS siblings with a heterozygous missense mutation in $\mathrm{CHD} 7$ were previously shown to also carry a heterozygous missense mutation in SEMA3A (40). Moreover, CHD7 haploinsufficiency affects the expression of Sema3a in mice (43).

To validate $S E M A 3 E$ as a novel KS gene and determine its role in the GnRH neuron system, we used in vitro models to demonstrate the pathogenicity of the R619C mutation and phenotyped KO mice lacking Sema3e or its receptor Plxnd1 or coreceptor $K d r$. These studies identified SEMA3E as a novel neurotrophic factor that signals via PLXND1 and KDR to activate prosurvival signaling in GnRH neurons. This function differs significantly from previously known roles for SEMA3E in modulating the cytoskeleton during vascular and axon guidance (44). Moreover, the functional importance of SEMA3E for neuron survival is fundamentally different from that of SEMA3A, which is mutated in other KS patients (7), but causes the ectopic migration of $\mathrm{GnRH}$ neurons along mispatterned axons in the nose (5). 
The p.R619C mutation we identified resides in the Ig-like C2-type domain that is conserved in all class 3 semaphorins. The basic domain of semaphorins enables binding to neuropilins with high affinity, and the signature SEMA domain confers specificity for neuropilins, which act as plexin coreceptors $(45,46)$. Instead, the Ig-like domain makes only a minor contribution to repulsive semaphorin activity in axon guidance (47), and its precise function has not previously been defined. Interestingly, the p.R619C mutation resides near a cysteine that enables dimerization of the Ig domain in other class 3 semaphorins (48). Computational modeling based on known crystal structures predicted that the novel cysteine residues are closely opposed in the mutant dimer and raises the possibility that an abnormal disulfide bridge might form and thereby impair structural flexibility. Together with the loss of a negative surface charge that may impact intra- or intermolecular interactions, this mutation was therefore predicted to compromise domain function. In agreement with this, functional assays demonstrated an essential role for SEMA3E in survival signaling via PI3 kinase activation that was impaired by the p.R619C mutation.

Notably, the SEMA3E prosurvival pathway we have identified is specific to GT1-7 cells, a model of developing GnRH neurons in the forebrain, but not GN11 cells, a model of GnRH neurons at an earlier stage of development, when they migrate in the nose. These observations agree with in vivo data on the role of VEGF/ NRP1 versus SEMA3E/PLXND1/KDR signaling in GnRH neuron development. Thus, immature GnRH neurons migrating in the embryonic nose express NRP1, and their pool is reduced in mice lacking NRP1 or NRP1-binding VEGF (21), but is shown here to be unaffected in mice lacking SEMA3E or PLXND1. By contrast, we found that GnRH neurons upregulate PLXND1 after forebrain entry and during their migration toward the hypothalamus and that they depend on SEMA3E/PLXND1 survival signaling at this developmental stage. This exquisite spatiotemporal specificity in signaling pathways for neurotrophic support appears an ideal adaption for neurons that have to negotiate molecularly distinct territories when traveling from their birthplace to the site of synaptogenesis.

An intriguing question is why developing GnRH neurons in the forebrain respond to SEMA3E with survival signaling rather than modulation of the actin cytoskeleton, as observed in endothelial cells of some vascular beds (9) and in axons (12). During axon guidance, cell type-specific responses to semaphorins are facilitated by coreceptor recruitment. Thus, PLXND1 signals axon attraction in neurons that coexpress NRP1, but repulsion in neurons lacking NRP1 (12). Moreover, the presence of KDR is required for SEMA3C-induced PI3K activation downstream of PLXND1/ NRP1 signaling for axonal growth in subico-mammillary neurons (11). On the basis of these observations, it appears likely that PLXND1 also recruits a coreceptor to activate PI3K in GnRH neurons and initiate the survival response. NRP1 is an unlikely cofactor for PLXND1 prosurvival signaling in GnRH neurons, because it is downregulated in these neurons after forebrain entry (A. Cariboni and C. Ruhrberg, unpublished observations). In contrast, VEGFR2 was expressed by GnRH neurons in the MPOA and was essential to maintain a normal number of these GnRH neurons in the brain, and, accordingly, the blockade of KDR signaling in GT1-7 cells compromised SEMA3E-mediated survival signaling. In contrast, KDR is not obviously expressed or required by GN11 cells or GnRH neurons migrating in the nose (21). Together, these observations suggest that GnRH neurons change their mechanism of survival signaling as they negotiate consecutive anatomical territories during their journey from the nasal placodes to the hypothalamus.

A role for PLXND1 in cell survival has recently been demonstrated in breast cancer cells, in which it acts as a dependence receptor that promotes cell death in the absence of the SEMA3E ligand. A role for SEMA3E as a rescue ligand to overcome the default pathway of receptor-induced death is, however, unlikely for GnRH neurons. First, the number of GnRH neurons is similarly reduced in mice lacking the PLXND1 receptor or the SEMA3E ligand, arguing against a role for PLXND1 in inducing GnRH neuron death. Second, we found that SEMA3E promoted GnRH neuron survival in vitro in a PLXND1-dependent fashion via PI3K/AKT, a conventional survival pathway that inhibits proapoptotic mediators such as BAX and BAD in neurons (27).

In conclusion, our study has uncovered a hitherto unknown role for SEMA3E as a neurotrophic factor that is essential for GnRH neuron development by signaling via PLXND1 to activate PI3K. Moreover, we have demonstrated that exome sequencing, combined with bioinformatics tools, tissue culture models, and the analysis of genetically modified mice, enables the identification and functional description of genes involved in GnRH neuron development and disease. We therefore anticipate that our study will prompt direct SEMA3E mutation screening in KS patients and lead to the increased application of exome sequencing for KS gene discovery as well as provide a starting point for mechanistic studies of GnRH neuron development and deficiency.

\section{Methods}

DNA sequencing and bioinformatics. Genomic DNA from 174 subjects (121 diagnosed with KS/HH, 53 unaffected) was prepared from white blood cells using a standard procedure and the exomes sequenced with an Illumina HiSeq 2000 platform using the Agilent V5 Exon Capture kit (Agilent Technologies). Exome sequencing alignment data for the 2 brothers affected by KS for SEMA3E, CHD7, and the known genes underlying $\mathrm{KS} / \mathrm{HH}$ are available at the European Nucleotide Archive (ENA) (accession number PRJEB8931). The p.R619C mutation in SEMA3E and the p.F1019C mutation in CHD7 were confirmed by Sanger sequencing. To calculate evolutionary constraint against nucleotide substitution at position c. $1855 \mathrm{C}>\mathrm{T}$ in SEMA3E, we used the GERP++ analysis tool.

Mouse strains. To obtain embryos of defined gestational stages, mice were mated in the evening, and the morning of vaginal plug formation was counted as E0.5. We used mice carrying Plxnd1-null, Sema3e-null, or Chd7-null (gift of Peter Scambler, UCL) alleles and mice carrying floxed conditional Plxnd1-null or $K d r$-null alleles with a Nes-Cre transgene $(9,11,29,30,41)$.

Immunostaining. Formaldehyde-fixed tissue sections and cells were incubated with PBS containing 10\% normal goat serum and $0.1 \%$ Triton X-100 or, for primary goat antibodies, with serum-free protein block (DAKO). The following primary antibodies were used for immunostaining: rabbit anti-peripherin (1:500; catalog AB1530; Chemicon); rabbit anti-GnRH (1:400; catalog 20075; ImmunoStar); rabbit anti-activated caspase-3; goat anti-human/mouse SEMA3E; and goat anti-human/mouse PLXND1 and goat anti-mouse KDR (1:150; catalogs MAB835, AF3239, AF4160, and AF644, respectively; 
R\&D Systems). Secondary antibodies used included Alexa Fluorconjugated goat anti-rabbit IgG (1:500; Life Technologies) and, in the case of primary goat antibodies, Cy3-conjugated donkey anti-goat Fab fragments (1:400; Jackson ImmunoResearch). For immunoperoxidase labeling, cryostat sections of formaldehyde-fixed samples were incubated with hydrogen peroxide to quench endogenous peroxidase activity before incubation with GnRH primary antibody (1:1,000), followed by biotinylated goat anti-rabbit antibody (1:400; Vector Laboratories), and then developed with the $\mathrm{ABC}$ kit (Vector Laboratories) and DAB (Sigma-Aldrich). Blood vessels were labeled with Alexa Fluor 488-conjugated isolectin B4 (IB4) (1:400) and nuclei counterstained with DAPI (Sigma-Aldrich). In some experiments, we performed ISH (49) for Gnrh (gift of Fani Memi, UCL) before immunostaining for activated caspase-3 or a TUNEL assay (ApopTag Red; EMD Millipore) before immunostaining for $\mathrm{GnRH}$.

Immunoblotting. Cells were lysed in $150 \mathrm{mM} \mathrm{NaCl}, 50 \mathrm{mM}$ Tris$\mathrm{HCl}(\mathrm{pH} 7.4)$, and $1 \%$ Triton $\mathrm{X}-100$, supplemented with protease and phosphatase inhibitors (Roche). Lysates were centrifuged at 10,000 $g$ for 10 minutes at $4^{\circ} \mathrm{C}$ and protein concentration determined with the Bradford assay (Bio-Rad). The conditioned media of transfected COS cells were concentrated by diafiltration with Microcon YH3O tubes (EMD Millipore) and diluted 1:2 in Laemmli sample buffer (24). Protein lysate $(20 \mu \mathrm{g})$ or concentrated conditioned media $(20 \mu \mathrm{l})$ were used for SDS-PAGE. Proteins were transferred to nitrocellulose membranes (Bio-Rad) and immunoblotted with rabbit anti-phosphorylated AKT (Ser473) and rabbit anti-AKT (1:1,000; catalogs 9271 and 9272, respectively; Cell Signaling Technology); goat anti-SEMA3E (1:500; catalog AF3239; R\&D Systems); mouse $\alpha$-tubulin (1:5,000; catalog T568; Sigma-Aldrich); followed by HRP-conjugated anti-rabbit, antigoat, or anti-mouse antibodies (1:10,000; catalogs sc-2301, sc-2020, and sc-2314; Santa Cruz Biotechnology Inc.).

AP fusion protein-binding assays. To introduce the p.R619C mutation into human SEMA3E, we mutated an expression vector containing AP-SEMA3E (12) using the QuickChange Lighting Kit (Agilent Technologies) and the oligonucleotides 5'-TCCTCTTTTCTTGTCTCACATCCTTTCTGTACAAACCAG-3' and 5'-CTGGTTTGTACAGAAAGGATGTGAGACAAGAAAAGAGGA-3' (Sigma-Aldrich). Human AP-SEMA3E proteins were prepared as previously described (50). GT1-7 cells were fixed for 5 minutes in methanol, washed 5 times with PBS, incubated in PBS containing $10 \%$ FBS for 30 minutes, and then reacted with AP fusion protein for 2 hours at room temperature. Cells were then washed for 5 minutes each with PBS, fixed with $4 \%$ formaldehyde for 2 minutes at room temperature, and washed again. Endogenous AP was heat inactivated by incubation at $65^{\circ} \mathrm{C}$ for 3 hours. Cell-bound, heat-stable recombinant AP activity was detected as an insoluble reaction product after incubation with nitro blue tetrazolium chloride and 5-bromo-4-chloro-3-indolyl phosphate (Roche).

Survival assays. GN11 or GT1-7 cells (gifts of Sally Radovick, Johns Hopkins University School of Medicine, Baltimore, Maryland, USA, and Pamela Mellon, UCSD, San Diego, California, USA, respectively) were seeded in 24-well plates at a density of 5,000 or 20,000 cells per well, respectively, and grown for 24 hours in DMEM containing 1 $\mathrm{mM}$ sodium pyruvate, $100 \mathrm{mg} / \mathrm{ml}$ streptomycin, $100 \mathrm{U} / \mathrm{ml}$ penicillin, and 10\% FBS (Life Technologies). GN11 and GT1-7 cells were serum starved for 48 and 24 hours, respectively, and then grown for a further 12 hours in serum-free medium in the absence or presence of $10 \mathrm{ng} / \mathrm{ml}$ recombinant human SEMA3E (R\&D Systems) or conditioned media of COS cells transfected with vectors encoding AP or AP-tagged human
SEMA3E. We determined the number of PI-stained cells relative to the total number of all Hoechst fluorochrome-positive cells. In some experiments, $1 \mathrm{mg} / \mathrm{ml}$ control IgG (R\&D Systems) or function-blocking antibodies (goat anti-PLXND1 or goat anti-KDR, see above) were added 1 hour before SEMA3E treatment. In other experiments, the PI3K inhibitor LY294 (Sigma-Aldrich) was added at $10 \mu \mathrm{M} 1$ hour prior to addition of SEMA3E. All assays were repeated in 3 independent experiments.

RT-PCR. Total RNA was extracted from GN11 and GT1-7 cells with TRIzol (Life Technologies). Single-stranded cDNA was synthesized with AMV reverse transcriptase and random hexamers (Promega). PCR was performed as previously described (51). PCR products were analyzed by electrophoresis on a $2 \%$ agarose gel and bands visualized under UV illumination after ethidium bromide staining. We used the following oligonucleotides: Plxnd1, 5'-TCCTAGACAGCCCTAACCCC-3' and 5'-AGGCTCAATCGCTCGGATTT-3'; $K d r, 5^{\prime}$-ACAGTAGCCGTCAAGATGTT-3' and 5'-CTACATCACTGAGCGATTTC-3'; and Gapdh, 5'-GGCCCCTCTGGAAAGCTGTGG-3' and 5'-TCTTGCTCAGTGTCCTTGCTGGG-3'.

Image processing. To acquire bright-field images, we used a Zeiss Axiovert 200 with a Photometrics CoolSNAP ES camera (survival assays) or a Leica DM microscope with a DC500 digital camera (AP assays, HRP-stained sections). We used a Leica TCS SPE1 confocal microscope to acquire fluorescence images of mouse tissues. All images were processed using Photoshop CS4 (Adobe Inc.). In some experiments, we acquired bright-field images of ISH patterns and confocal images of immunofluorescence staining and converted the bright-field images to RGB color mode for superimposition onto the confocal images.

Quantitation. To determine the total number of GnRH neurons in E14.5 mouse heads, E17.5 mouse brains, or adult mouse brains, $25-\mu \mathrm{m}$ sagittal sections through the entire head/brain of 3 or more animals for each genotype were immunolabeled for $\mathrm{GnRH}$ and all GnRH-positive cells counted in each section, as previously described (5). We determined the number of apoptotic cells as the average of all cells positive for activated caspase-3 in 3 adjacent $20-\mu \mathrm{m}$ sagittal sections through the brain at the level of the GnRH neuron-containing MPOA in 3 independent experiments. To compare the abundance of GnRH-positive neurites at the ME in WT and mutant brains, we measured the pixel intensity of GnRH staining in 20- $\mu \mathrm{m}$ sagittal sections through the ME of 3 mice for each genotype. We quantified the number of $\mathrm{GnRH}$ and PLXND1 double-positive cells in all GnRH-positive cells in $20-\mu \mathrm{m}$ coronal sections of the MPOA as the average of 3 sections in each of 3 embryos. For survival assays, we determined the percentage of PIpositive cells in all Hoechst-positive GN11 or GT1-7 cells for each treatment group in 12 random fields from 3 independent experiments. For immunoblotting, 3 independent experiments were performed for each condition, the OD of the signal measured with ImageJ software (NIH), and the mean pixel intensity calculated.

Statistics. For all experiments, data are expressed as the mean \pm SEM. To determine statistical significance, we used the unpaired $t$ test or, for multiple comparisons, a 1-way ANOVA followed by a Dunnett's post-hoc test. A $P$ value of less than 0.05 was considered statistically significant. Statistical analysis was performed using GraphPad Prism4 (GraphPad Software).

Study approval. All animal experiments were conducted with approval from the Animal Welfare and Ethical Review Body of the UCL Institute of Ophthalmology and carried our under UK Home Office licence 70/7126. 
Blood samples were obtained from patients at the Royal Free Hospital with their explicit consent for extracting DNA for mutational analysis.

\section{Acknowledgments}

We are grateful to Nelly Pitteloud and James Acierno Jr. for help with exome sequencing and analysis. We are also grateful to John Parnavelas and Roberto Maggi for access to equipment and reagents. We thank Sally Radovick and Pamela Mellon for the GN11 and GT1-7 cells, respectively, Peter Scambler for the Chd7-mutant mouse strain, and Fani Memi for the Gnrh riboprobe. We thank Claudio Raimondi, Andy Joyce, Sonja Rakic, Emanuele Sala, Matteo Paxia, and Roberto Oleari for technical assistance and the staff of the Biological Resources Unit of the UCL Institute of Ophthalmology and the Institut de Biologie du Développement de Marseille (IBDM) for help with mouse husbandry.
This research was funded by grants from the Biotechnology and Biological Sciences Research Council (BBSRC) (BB/L002639/1) and the Wellcome Trust (08977/Z/09/Z), to C. Ruhrberg; from Telethon (GGP13142), to A. Cariboni; and from the Agence Nationale de la Recherche (ANR) (ANR-10-BLANC-1412-02), to F. Mann. The funders had no role in study design, data collection and analysis, decision to publish, or preparation of the manuscript.

Address correspondence to: Christiana Ruhrberg, UCL Institute of Ophthalmology, 11-43 Bath Street, London EC1V 9EL, United Kingdom. Phone: 44.20.7608.4017; E-mail: c.ruhrberg @ucl.ac.uk. Or to: Anna Cariboni, University of Milan, Department of Pharmacological and Biomolecular Sciences, Via Balzaretti 9, 20133 Milan, Italy. Phone: 39.02.503.18230; E-mail: anna.cariboni@unimi.it.
1. Wierman ME, Kiseljak-Vassiliades K, Tobet S. Gonadotropin-releasing hormone (GnRH) neuron migration: initiation, maintenance and cessation as critical steps to ensure normal reproductive function. Front Neuroendocrinol. 2011;32(1):43-52.

2. Sykiotis GP, Pitteloud N, Seminara SB, Kaiser UB, Crowley WF Jr. Deciphering genetic disease in the genomic era: the model of GnRH deficiency. Sci Transl Med. 2010;2(32):32rv2.

3. Schwanzel-Fukuda M, Bick D, Pfaff DW. Luteinizing hormone-releasing hormone (LHRH)expressing cells do not migrate normally in an inherited hypogonadal (Kallmann) syndrome. Brain Res Mol Brain Res. 1989;6(4):311-326.

4. Wray S. From nose to brain: development of gonadotrophin-releasing hormone-1 neurones. J Neuroendocrinol. 2010;22(7):743-753.

5. Cariboni A, Davidson K, Rakic S, Maggi R, Parnavelas JG, Ruhrberg C. Defective gonadotropin-releasing hormone neuron migration in mice lacking SEMA3A signalling through NRP1 and NRP2: implications for the aetiology of hypogonadotropic hypogonadism. Hum Mol Genet. 2011;20(2):336-344.

6. Young J, et al. SEMA3A deletion in a family with Kallmann syndrome validates the role of semaphorin $3 \mathrm{~A}$ in human puberty and olfactory system development. Hum Reprod. 2012;27(5):1460-1465.

7. Hanchate NK, et al. SEMA3A, a gene involved in axonal pathfinding, is mutated in patients with Kallmann syndrome. PLoS Genet. 2012;8(8):e1002896.

8. Pitteloud N, Durrani S, Raivio T, Sykiotis GP. Complex genetics in idiopathic hypogonadotropic hypogonadism. Front Horm Res. 2010;39:142-153.

9. $\mathrm{Gu}$ C, et al. Semaphorin $3 \mathrm{E}$ and plexin-D1 control vascular pattern independently of neuropilins. Science. 2005;307(5707):265-268.

10. Kim J, Oh WJ, Gaiano N, Yoshida Y, Gu C. Semaphorin 3E-Plexin-D1 signaling regulates VEGF function in developmental angiogenesis via a feedback mechanism. Genes Dev. 2011;25(13):1399-1411.

11. Bellon A, et al. VEGFR2 (KDR/Flk1) signaling mediates axon growth in response to semaphorin $3 \mathrm{E}$ in the developing brain. Neuron.
2010;66(2):205-219.

12. Chauvet S, et al. Gating of Sema3E/PlexinD1 signaling by neuropilin-1 switches axonal repulsion to attraction during brain development. Neuron. 2007;56(5):807-822.

13. Ding JB, Oh WJ, Sabatini BL, Gu C. Semaphorin 3E-Plexin-D1 signaling controls pathway-specific synapse formation in the striatum. Nat Neurosci. 2012;15(2):215-223.

14. Kumar P, Henikoff S, Ng PC. Predicting the effects of coding non-synonymous variants on protein function using the SIFT algorithm. Nat Protoc. 2009;4(7):1073-1081.

15. Adzhubei IA, et al. A method and server for predicting damaging missense mutations. Nat Methods. 2010;7(4):248-249.

16. Schwarz JM, Rodelsperger C, Schuelke M, Seelow D. MutationTaster evaluates disease-causing potential of sequence alterations. Nat Methods. 2010;7(8):575-576.

17. Davydov EV, Goode DL, Sirota M, Cooper GM, Sidow A, Batzoglou S. Identifying a high fraction of the human genome to be under selective constraint using GERP++. PLoS Comput Biol. 2010;6(12):e1001025.

18. Kim DE, Chivian D, Baker D. Protein structure prediction and analysis using the Robetta server. Nucleic Acids Research. 2004;32(Web Server issue):W526-W531.

19. Maggi R, Pimpinelli F, Molteni L, Milani M, Martini L, Piva F. Immortalized luteinizing hormone-releasing hormone neurons show a different migratory activity in vitro. Endocrinology. 2000;141(6):2105-2112.

20. Giacobini P, et al. Hepatocyte growth factor acts as a motogen and guidance signal for gonadotropin hormone-releasing hormone-1 neuronal migration. J Neurosci. 2007;27(2):431-445.

21. Cariboni A, et al. VEGF signalling controls GnRH neuron survival via NRP1 independently of KDR and blood vessels. Development. 2011;138(17):3723-3733.

22. Zhang $\mathrm{G}$, et al. Hypothalamic programming of systemic ageing involving IKK- $\beta, \mathrm{NF}-\kappa \mathrm{B}$, and GnRH. Nature. 2013;497(7448):211-216.

23. Cariboni A, Rakic S, Liapi A, Maggi R, Goffinet A, Parnavelas J. Reelin provides an inhibitory signal in the migration of gonadotropin-releasing hormone neurons. Development. 2005;132(21):4709-4718.

24. Cariboni A, et al. The product of X-linked Kallmann's syndrome gene (KAL1) affects the migratory activity of gonadotropin-releasing hormone (GnRH)-producing neurons. Hum Mol Genet. 2004;13(22):2781-2791.

25. Luchino J, et al. Semaphorin $3 \mathrm{E}$ suppresses tumor cell death triggered by the plexin D1 dependence receptor in metastatic breast cancers. Cancer Cell. 2013;24(5):673-685.

26. Sakurai A, et al. Semaphorin 3E initiates antiangiogenic signaling through plexin D1 by regulating Arf6 and R-Ras. Mol Cell Biol. 2010;30(12):3086-3098.

27. Brunet A, Datta SR, Greenberg ME. Transcription-dependent and -independent control of neuronal survival by the PI3K-Akt signaling pathway. Curr Opin Neurobiol. 2001;11(3):297-305.

28. Bortner CD, Oldenburg NB, Cidlowski JA. The role of DNA fragmentation in apoptosis. Trends Cell Biol. 1995;5(1):21-26.

29. Zhang Y, et al. Tie2Cre-mediated inactivation of plexinD1 results in congenital heart, vascular and skeletal defects. Dev Biol. 2009;325(1):82-93.

30. Tronche F, et al. Disruption of the glucocorticoid receptor gene in the nervous system results in reduced anxiety. Nat Genet. 1999;23(1):99-103.

31. Gitler AD, Lu MM, Epstein JA. PlexinD1 and semaphorin signaling are required in endothelial cells for cardiovascular development. Dev Cell. 2004;7(1):107-116.

32. Pitteloud N, et al. Digenic mutations account for variable phenotypes in idiopathic hypogonadotropic hypogonadism. J Clin Invest. 2007;117(2):457-463.

33. Dode C, et al. Kallmann syndrome: mutations in the genes encoding prokineticin-2 and prokineticin receptor-2. PLoS Genet. 2006;2(10):e175.

34. Cole LW, et al. Mutations in prokineticin 2 and prokineticin receptor 2 genes in human gonadotrophin-releasing hormone deficiency: molecular genetics and clinical spectrum. JClin Endocrinol Metab. 2008;93(9):3551-3559.

35. Miura K, Acierno JJ, Seminara S. Characterization of the human nasal embryonic LHRH factor gene, NELF, and a mutation screening among 65 patients with idiopathic hypogonadotropic hypogonadism (IHH).JHum Genet. 2004;49(5):265-268. 
36. Canto P, Munguia P, Soderlund D, Castro JJ, Mendez JP. Genetic analysis in patients with Kallmann syndrome: coexistence of mutations in prokineticin receptor 2 and KAL1. J Androl. 2009;30(1):41-45.

37. Bajpai R, et al. CHD7 cooperates with PBAF to control multipotent neural crest formation. Nature. 2010;463(7283):958-962.

38. Engelen E, et al. Sox 2 cooperates with Chd7 to regulate genes that are mutated in human syndromes. Nat Genet. 2011;43(6):607-611.

39. Bergman JE, et al. The results of CHD7 analysis in clinically well-characterized patients with Kallmann syndrome. JClin Endocrinol Metab. 2012;97(5):E858-E862.

40. Marcos S, et al. The prevalence of CHD7 missense versus truncating mutations is higher in patients with Kallmann syndrome than in typical CHARGE patients. JClin Endocrinol Metab. 2014;99(10):E2138-E2143.

41. Jones KM, Saric N, Russell JP, Andoniadou CL, Scambler PJ, Basson MA. CHD7 maintains neu- ral stem cell quiescence and prevents premature stem cell depletion in the adult hippocampus. Stem Cells. 2015;33(1):196-210.

42. Bergman JE, Bosman EA, van Ravenswaaij-Arts CM, Steel KP. Study of smell and reproductive organs in a mouse model for CHARGE syndrome. Eur JHum Genet. 2010;18(2):171-177.

43. Schulz Y, et al. CHD7, the gene mutated in CHARGE syndrome, regulates genes involved in neural crest cell guidance. Hum Genet. 2014;133(8):997-1009.

44. Oh WJ, Gu C. The role and mechanism-of-action of Sema3E and Plexin-D1 in vascular and neural development. Seminars in Cell \& Dev Biol. 2013;24(3):156-162.

45. Feiner L, Koppel AM, Kobayashi H, Raper JA. Secreted chick semaphorins bind recombinant neuropilin with similar affinities but bind different subsets of neurons in situ. Neuron. 1997;19(3):539-545

46. He Z, Tessier-Lavigne M. Neuropilin is a receptor for the axonal chemorepellent Semaphorin III.
Cell. 1997;90(4):739-751.

47. Koppel AM, Feiner L, Kobayashi H, Raper JA. A 70 amino acid region within the semaphorin domain activates specific cellular response of semaphorin family members. Neuron. 1997;19(3):531-537.

48. Koppel AM, Raper JA. Collapsin-1 covalently dimerizes, and dimerization is necessary for collapsing activity.J Biol Chem. 1998;273(25):15708-15713.

49. Memi F, Abe P, Cariboni A, MacKay F, Parnavelas JG, Stumm R. CXC chemokine receptor 7 (CXCR7) affects the migration of GnRH neurons by regulating CXCL12 availability. J Neurosci. 2013;33(44):17527-17537.

50. Vieira JM, Schwarz Q, Ruhrberg C. Selective requirements for NRP1 ligands during neurovascular patterning. Development. 2007;134(10):1833-1843.

51. Cariboni A, et al. Neuropilins and their ligands are important in the migration of gonadotropin-releasing hormone neurons. J Neurosci. 2007;27(9):2387-2395. 